NBER WORKING PAPER SERIES

\title{
POLITICAL COMPETITION WITH CAMPAIGN CONTRIBUTIONS AND INFORMATIVE ADVERTISING
}

\author{
Stephen Coate \\ Working Paper 8693 \\ http://www.nber.org/papers/w8693 \\ NATIONAL BUREAU OF ECONOMIC RESEARCH \\ 1050 Massachusetts Avenue \\ Cambridge, MA 02138 \\ December 2001
}

Work on this project was undertaken during a visit to the Institute for International Economic Studies at the University of Stockholm. I thank the Institute for its hospitality and David Stromberg for useful discussions. I also thank Tim Besley for his input in the early stages of this project and Julia Epifantseva for excellent research assistance. The views expressed herein are those of the author and not necessarily those of the National Bureau of Economic Research.

(C) 2001 by Stephen Coate. All rights reserved. Short sections of text, not to exceed two paragraphs, may be quoted without explicit permission provided that full credit, including $\mathbb{C}$ notice, is given to the source. 
Political Competition with Campaign Contributions and Informative Advertising Stephen Coate

NBER Working Paper No. 8693

December 2001

JEL No. D72

\begin{abstract}
$\underline{\text { ABSTRACT }}$
This paper presents a model of political competition with campaign contributions and informative political advertising. Policy-motivated parties compete by selecting candidates and interest groups provide contributions to enhance the electoral prospects of like-minded candidates. Contributions are used to finance advertising campaigns that provide voters with information about candidates' ideologies. The model embodies rational behavior on the part of all actors, is analytically tractable, and has a unique equilibrium. The paper uses the model to analyze the welfare economics of contribution limits. Such limits are shown to redistribute welfare from moderate voters to interest group members. They may or may not raise aggregate welfare.
\end{abstract}

\author{
Stephen Coate \\ Department of Economics \\ Cornell University \\ Ithaca, NY 14853 \\ and NBER \\ sc163@cornell.edu
}




\section{Introduction}

This paper presents a model of political competition with campaign contributions and informative political advertising. The model incorporates three groups of political actors; parties who select candidates, interest groups who contribute to candidates, and voters who vote for candidates. Campaign contributions are position induced in the sense that interest groups give to enhance the electoral prospects of like-minded candidates (as opposed to buying policy favors). Interest groups' contributions are used to finance advertising campaigns that provide voters with information about candidates' ideologies. The model embodies rational behavior on the part of all actors, is analytically tractable, and has a unique equilibrium.

In light of the explosion of theoretical models of electoral competition, the reader might be forgiven for questioning the value of yet another one. ${ }^{1}$ The development of this model is motivated by a desire to analyze issues in the financing of political campaigns. The appropriate regulation of campaign contributions is an important topic in the United States, with significant dissatisfaction with the current system and many proposals for reform. To analyze such proposals, one needs a framework that adequately captures the role contributions play in elections. As commonly understood, this role is to finance the provision of information about candidates to voters. From a social viewpoint, this means that contributions should help produce more informed choices. Moreover, their presence should provide parties with a greater incentive to select candidates with characteristics that voters want. While there have been a number of interesting studies of campaign contributions, the literature has yet to produce a theory that satisfactorily captures this role. The model presented here is designed to fill this gap.

The model assumes that there are two political parties representing opposing ideologies. Parties can choose to run either partisan or moderate candidates, with party members preferring the

\footnotetext{
${ }^{1}$ See Persson and Tabellini (2000) and Grossman and Helpman (2001) for overviews of recent work.
} 
ideology of the former. Voters know a candidate's party affiliation but not whether he is a partisan or a moderate. Advertising allows a candidate to provide voters with this information. The idea is that candidates have past records that reveal their ideologies and advertising can inform voters of those records. ${ }^{2} \quad$ Such advertising can be advantageous for a moderate candidate because it may attract moderate voters of the opposing ideology. Resources for campaign advertising are provided by interest groups consisting of partisans of opposing ideologies. These groups contribute only to moderate candidates because only they can benefit from advertising their ideologies. ${ }^{3}$

To illustrate the value of the model for policy analysis, the paper uses it to study the case for limiting the amount that interest groups can contribute to campaigns. The analysis reveals a basic trade-off: contribution limits reduce expected campaign spending, but also decrease the likelihood that parties select moderate candidates. The existence of this trade-off means that contribution limits cannot be evaluated via the Pareto criterion. Rather, they redistribute welfare between different groups. Moderate voters are made worse off; partisans who do not belong to interest groups are unaffected; and partisans who are interest group members are actually made better off. Thus, imposing a contribution limit redistributes welfare from moderates to interest group members. The question of whether the gains to interest group members offset the losses to moderate voters turns on the fraction of the latter in the population. Contribution limits are always desirable from an aggregate viewpoint when only a small fraction of the population are moderates, while limits will tend to be harmful when moderates dominate.

The organization of the remainder of the paper is as follows. The next section explains how

\footnotetext{
2 Thus, for example, moderate left-wing candidates will have records of being tough on crime, fiscally responsible, or pro vouchers. Alternatively, if they have not previously held elected office, they will have had business experience and displayed strong family values. In contrast, their partisan counterparts will have records of support for radical programs (such as gay marriage), a history of community activism, or strong ties to the union movement. They may also have belonged to partisan associations (such as Al Sharpton's presidential advisory committee).

3 The conclusion that partisan candidates recieve no campaign contributions arises because there is only one dimension of difference between candidates and negative advertising is not permitted. In reality, it would be an unusual race in which a candidate could not benefit by revealing some information about himself or his opponent. Nonetheless, the general conclusion that those candidates with characteristics that swing voters value should, ceteris paribus, receive larger contributions would seem a natural implication of the informational perspective.
} 
the paper relates to previous work on electoral competition and campaign contributions, and to the literature on informative advertising in market contexts. Section 3 presents the model and Section 4 studies equilibrium. Section 5 analyzes the impact of contribution limits. Section 6 concludes with a summary of the lessons of the analysis and some suggestions for future research.

\section{Related literature}

The model presented here builds on the work of Besley and Coate (2000) and (2001). These papers employ a model of political competition in which policy-motivated parties compete by selecting candidates who are characterized by their policy preferences and interest groups provide position induced contributions to these candidates. ${ }^{4}$ In the model, however, contributions are used to finance advertising that attracts the votes of "noise voters". 5 The reason why noise voters respond to advertising is not explained. Moreover, a candidate's ability to attract their votes is independent of his or his opponent's policy stance, precluding an informational interpretation. This paper extends this model of electoral competition to incorporate an informational, microfounded role for campaign contributions.

There are relatively few papers that study informative campaign advertising. Austen-Smith (1987) presents a framework that shares some features with that presented here. In his model, two office-seeking candidates compete by selecting positions in a one dimensional policy space. Two interest groups with ideal points to the left and right of the median voter offer position induced contributions. Voters have common but noisy perceptions of candidates' policy positions and campaign advertising reduces the variance of these perceptions. Since voters are risk averse, this helps even a candidate with an extreme position. The focus of the analysis is to understand how the presence of the interest groups influences the positions taken by the candidates.

\footnotetext{
4 These papers in turn build on the citizen-candidate models of Besley and Coate (1997) and Osborne and Slivinski (1996).

5 This follows the approach of Baron (1994) and Grossman and Helpman (1996).
} 
While interesting and creative, Austen-Smith's analysis has two drawbacks. First, it is not explained why voters are uncertain about candidates' policy positions and how this uncertainty is reduced by advertising. Indeed, in equilibrium, there is no uncertainty in candidates' choices and hence, from a game theoretic perspective, no reason that voters should be uncertain. Second, without artificially restricting the candidates to choose different positions, equilibrium involves candidates choosing the same positions and no contributions being given. Thus, the model does not naturally yield an explanation for campaign contributions.

Ortuno-Ortin and Schultz (2000) discuss informative advertising in their interesting analysis of the public funding of campaigns. They model two policy-motivated political parties who compete by choosing positions in a one-dimensional policy space. There is a fixed pot of public money made available for financing campaigns. The two parties must use any money allocated to them to inform voters of their positions. ${ }^{6}$ The paper studies parties' choice of platforms under two assumptions concerning the allocation of public funding: (i) each party receives an exogenous share of the funds and (ii) each party's share of the funds is an increasing function of its vote share. In equilibrium, there is no uncertainty concerning each party's platform choice and hence all voters, having been exposed to advertising or not, know each party's position. This feature means that the campaign advertising is ineffective in equilibrium. Accordingly, if it were financed by private citizens, none would be provided.

Schultz (2001) discusses the targeting of informative advertising in a model in which two parties compete for votes by promising transfers to different groups (as in Lindbeck and Weibull (1987)). Parties care both about votes and the ideological purity of their proposed redistributions. Voters do not directly observe parties' proposals but can be informed of their transfers via campaign advertising. Each party has an exogenous advertising budget and advertising can be perfectly

\footnotetext{
6 If voters believe that their position is closer to the median than it actually is, then parties would have no incentive to advertise and hence this constraint may bind.
} 
targeted to the different groups. Voters are uncertain about parties' ideological preferences so that, in equilibrium, there is genuine uncertainty concerning parties' proposals. As in this paper, uninformed voters make rational inferences based on knowledge of parties' strategies. Schultz studies the joint determination of transfers and advertising, establishing a number of interesting conclusions concerning the groups who will attract the most intensive campaigning. Schultz's analysis is complementary to that pursued here in that it seeks to shed light on how parties will use such campaign resources as they have. By contrast, the analysis of this paper seeks to understand the level of resources allocated to campaigning.

Following the industrial organization literature on advertising as a signal (Milgrom and Roberts (1986)), a number of authors have argued that campaign advertising is best understood as providing information indirectly (Potters, Sloof, and Van Winden (1997), Prat (1999), (2000)). These papers assume that candidates have some valence characteristic such as competence and interest groups can observe this more precisely than voters. The amount of campaign money that a candidate collects signals his valence to voters. Hence, although campaign advertising does not convey information directly, it does so indirectly. In Prat's (1999) insightful analysis, two office-seeking candidates, who may differ in competence, compete by staking out positions in a one dimensional policy space. A single interest group with non-median policy preferences offers contributions to candidates in exchange for them moving their platforms towards its preferred policy position. Thus contributions are service induced. Candidates the interest group believes to be more competent are offered larger contributions because they are more likely to win. This is because voters observe a noisy signal of competence and hence, ceteris paribus, are more likely to vote for the more competent candidate. In equilibrium, therefore, the more a candidate advertises, the higher is his competence. Campaign contributions are good for voters in the sense that they provide information about competence, but bad in that they lead candidates to distort policy. Banning contributions can raise voters' aggregate welfare when the losses in terms of information about 
competence are smaller than the costs of policy distortion. ${ }^{7}$

There is much of interest in this argument. However, the widespread employment of high priced political strategists suggests that the content of campaign advertisements matters. ${ }^{8}$ Moreover, campaign advertisements typically do provide verifiable information about candidates' records. Such information includes their accomplishments in the private sector or military service, their family history, the public programs they have proposed or supported, things they have done for their constituents, endorsements from community leaders, etc. While it is true that there is nothing in the United States legal system to prevent candidates from lying about these things, ${ }^{9}$ the threat of being exposed by the media (or perhaps just basic human decency) makes bold-faced lying the exception rather than the rule. Thus, to focus solely on the signalling role of campaign advertising misses an important part of its function.

It is interesting to contrast the role of informative advertising in political competition with that in market competition (see, for example, Nelson (1976)). In political competition, one may think of the candidates that parties select as differentiated products and an individual voter's decision to vote for a particular candidate as like a purchase decision. To the extent that interest groups seek to maximize the votes for their party's candidate, the situation seems broadly analogous to informative advertising by producers in an industry with differentiated products. The seminal analysis of such advertising is due to Grossman and Shapiro (1984). In their model, firms are characterized by their product type and use advertising to inform consumers of their product and

\footnotetext{
7 See Prat (1999) for the precise conditions under which this occurs. While Prat does not consider the distributional consequences of contribution limits, it seems likely that in his model banning contributions is either Pareto inefficient or redistributes from citizens on the side of the interest group to those on the other side of the political spectrum. Welfare conclusions more in keeping with the findings of this paper might be expected with two interest groups with preferred policies either side of the median voter. In such circumstances, interest group competition may reduce the costs of policy distortion and thereby dissipate interest groups' policy gains. For an analysis of multiple interest groups in a related framework see Prat (2000).

8 Presumably, it is unreasonable to suppose that voters observe the wage paid to political consultants and hence the hiring of such consultants cannot itself be put down to signalling.

${ }^{9}$ In the United States, the First Amendment protects advertising as free speech. Thus, in contrast to commercial advertising, citizens have no legal recourse against a candidate who lies about his (or his opponent's) record.
} 
its price. Consumers choose from the set of products whose advertisements they see. Accordingly, if a firm does not advertise it receives no sales because consumers are unaware of its existence. Thus, advertising plays the social role of creating surplus enhancing matches. With respect to the optimality of the equilibrium level of advertising, Grossman and Shapiro identify two conflicting divergences between private and social incentives. On the one hand, firms under-advertise because they do not capture all the surplus from the matches they create. On the other, they over-advertise because they do not take into account the fact that some of their increased sales come at the expense of competitors.

Contrasting the analysis here with Grossman and Shapiro (1984) suggests at least three differences between informative advertising in political and market competition. First, and most fundamentally, voters see a list of all candidates at the time they vote and hence must be aware of the existence of a candidate even if they have not seen this candidate's advertisement. Thus, if they do not know a candidate's characteristics, they must form expectations of them at the time of voting. These expectations determine the effectiveness of campaign advertising. For if voters expect unadvertised candidates to have the attributes of advertised candidates, campaign advertising will be ineffective. The necessity of solving for citizens' beliefs concerning unadvertised candidates is the major analytical challenge in studying advertising in the political context. A second difference is that parties choose their candidates - they do not inherit fixed locations. ${ }^{10}$ Finally, since parties choose candidates and interest groups finance them, the actors choosing product characteristics and those financing their advertising are different. Moreover, interest groups are concerned with the policy outcome and hence are concerned with votes only indirectly. These differences not withstanding, analogies to the divergences between private and social incentives leading to over and under advertising in the market context emerge from this analysis.

\footnotetext{
10 This is a difference between the analysis in this paper and that in Grossman and Shapiro (1984). Obviously, in reality, firms choose the type of their products as well.
} 


\section{The model}

\subsection{Basics}

A community must elect a representative. Citizens differ in their ideology which is measured on a 0 to 1 scale. The population is divided into three groups: leftists, rightists, and moderates. Leftists and rightists have ideologies $d$ and $1-d$ respectively, where $d<\frac{1}{2}$. Moderates come in two types: left-leaning and right-leaning with ideologies $x$ and $1-x$ where $x \in\left(d, \frac{1}{2}\right)$. Moderates make up a fraction $\gamma$ of the community and the remaining $1-\gamma$ are evenly divided between leftists and rightists. The fraction of moderates who are left-leaning, denoted $\mu$, is ex ante uncertain, reflecting the fluid nature of moderate attitudes. Specifically, $\mu$ is the realization of a random variable uniformly distributed on $[0,1]$.

Candidates for community representative are put forward by two political parties: Party $L$, comprised of leftists and Party $R$, comprised of rightists. Candidates are citizens and hence are characterized by their ideologies. Each party can select either a "partisan" or a "moderate" candidate. Specifically, Party $L$ selects a candidate with ideology $d$ or $x$ and Party $R$ chooses one with ideology $1-x$ or $1-d$. Decisions as to which type of candidate to select are made to maximize the payoff of the representative party member. At the time at which candidates are selected, party members do not know the fraction of moderates who are left-leaning making election outcomes uncertain.

The payoff enjoyed by a citizen with ideology $i$ from having a leader of ideology $i^{\prime}$ is given by $-\beta\left|i-i^{\prime}\right|$ where $\left|i-i^{\prime}\right|$ is the distance from $i$ to $i^{\prime}$ and $\beta>0$. It is assumed that $x-d>1-2 x$ which implies that moderates prefer a moderate candidate of the opposing ideology to a partisan candidate of their own ideology. Thus, left-leaning moderates (for example) will prefer the ideology of Party R's candidate to that of Party L's candidate if the former is a moderate and the latter is a partisan. 
Moderates do not have perfect information about candidates, in the sense of not knowing whether each party's candidate is a partisan or a moderate. Such information could be acquired, but moderates are not politically engaged and choose to remain "rationally ignorant". However, candidates can convey information concerning their characteristics via advertising. Moderates cannot ignore such advertising since it is bundled with radio or television programming.

Campaign advertising is governed by the following rules. First, candidates can only advertise their own characteristics; i.e., whether they are moderate or partisan. This rules out negative advertising. ${ }^{11}$ Second, candidates can only advertise the truth. The idea is that candidates have records which reveal their ideologies and that candidates cannot lie about their records. The advertising technology is such that if a candidate spends an amount $C$, his message reaches a fraction $\lambda(C)=\frac{C}{\alpha+C}$ of the population, where $\alpha>0$.

Candidates' advertising is financed by campaign contributions provided by interest groups. There are two interest groups - a leftist group that contributes to Party $L$ 's candidate and a rightist group that contributes to Party $R$ 's. A fraction $\theta$ of partisans belong to each interest group. The interest groups choose contribution levels to maximize the expected payoff of their members. They observe the type of their party's candidate before making contributions, and hence only give to moderate candidates. It is assumed, however, that they do not observe the type of the opposing party's candidate at the time of contributing. ${ }^{12}$

Parties choose candidates anticipating the contributions they will receive and the impact of these on voters' choices. Each interest group observes its party's choice and decides on its con-

\footnotetext{
11 It would be possible to introduce negative advertising by allowing a candidate to inform voters that his opponent is a partisan. Since this significantly complicates the model, I leave it for future work.

12 This assumption is made to simplify the analysis. If interest groups know the type of the opposing party's candidate, they will contribute more to a candidate running against a partisan than a moderate. This is because the benefit to them of electing their own party's candidate is higher in the former case. This difference in contribution levels means that seeing an advertisement for a candidate provides information to voters about the likely type of his opponent. After all, a voter is more likely to see an advertisement for a candidate when he is running against a partisan. While this effect is interesting and it is perfectly possible to carry out the analysis taking it into account, it is an additional wrinkle that complicates an already intricate analysis. Accordingly, the effect is assumed away here.
} 
tribution. Partisans always vote for the candidate put forward by the party representing their ideology. Moderates, having possibly observed one or both candidates' advertisements, update their beliefs about candidates' types and vote. They may vote for either party's candidate. To smooth out behavior among the two types of moderates, they are assumed to vote probabilistically. These behaviors are now described in greater detail.

\subsection{Behavior of moderate voters}

At the time of voting, each moderate voter may have seen advertisements from both, one, or neither candidate. Let $\left(I_{L}, I_{R}\right)$ denote a moderate voter's information where $I_{K}=1$ if he has seen an advertisement from Party $K$ 's candidate and $I_{K}=0$ if not. Let $\rho_{K}\left(I_{L}, I_{R}\right)$ denote his belief that Party $K$ 's candidate is moderate conditional on informational state $\left(I_{L}, I_{R}\right)$. Since only moderate candidates advertise, both $\rho_{L}\left(1, I_{R}\right)$ and $\rho_{R}\left(I_{L}, 1\right)$ must equal 1 . The beliefs $\rho_{L}\left(0, I_{R}\right)$ and $\rho_{R}\left(I_{L}, 0\right)$ will be derived as part of the equilibrium. Letting $v_{K}\left(J ; I_{L}, I_{R}\right)$ denote a moderate voter of type $K$ 's expected payoff from Party $J$ 's candidate being elected when the voter has information $\left(I_{L}, I_{R}\right)$, we have that

$$
v_{K}\left(K ; I_{L}, I_{R}\right)=-\left(1-\rho_{K}\left(I_{L}, I_{R}\right)\right) \beta(x-d),
$$

and for $J \neq K$

$$
v_{K}\left(J ; I_{L}, I_{R}\right)=-\rho_{J}\left(I_{L}, I_{R}\right) \beta(1-2 x)-\left(1-\rho_{J}\left(I_{L}, I_{R}\right)\right) \beta(1-d-x) .
$$

Following the literature on probabilistic voting, a moderate voter of type $K$ in informational state $\left(I_{L}, I_{R}\right)$ votes for Party $L$ 's candidate if and only if $v_{K}\left(L ; I_{L}, I_{R}\right)+\varepsilon \geq v_{K}\left(R ; I_{L}, I_{R}\right)$ where $\varepsilon$ is the realization of a random variable with range $[-\bar{\varepsilon}, \bar{\varepsilon}](\bar{\varepsilon}>0)$ and symmetric cumulative distribution function $H(\varepsilon)$. The fraction of moderate voters of type $K$ in informational state $\left(I_{L}, I_{R}\right)$ voting for Party $L$ 's candidate is therefore

$$
\xi_{K}\left(I_{L}, I_{R}\right)=1-H\left(v_{K}\left(R ; I_{L}, I_{R}\right)-v_{K}\left(L ; I_{L}, I_{R}\right)\right),
$$


where we adopt the convention that $H(\varepsilon)=0$ for all $\varepsilon \leq-\bar{\varepsilon}$ and $H(\varepsilon)=1$ for all $\varepsilon \geq \bar{\varepsilon}$.

It is assumed that $\bar{\varepsilon}$ is smaller than the minimum of $\beta(1-2 x)$ and $\beta(3 x-d-1)$. This implies that type $K$ moderates who believe that Party $K$ 's candidate is at least as likely to be moderate as the opposing Party's candidate will always vote for him. In particular, this means that $\xi_{L}\left(1, I_{R}\right)=1$ and $\xi_{R}\left(I_{L}, 1\right)=0$. In addition, the assumption implies that all type $K$ moderates who know that their party's candidate is partisan and that the opposing candidate is moderate will vote for the latter.

\subsection{Election probabilities}

Given this voting behavior, the probability that each party's candidate will win may be computed. Let $C_{L}$ and $C_{R}$ denote the contribution levels the two interest groups provide to moderate candidates. Then, if both candidates are moderates, the fraction of left-leaning moderates voting for Party L's candidate is

$$
\delta_{L}\left(C_{L}, C_{R}\right)=\lambda\left(C_{L}\right)+\xi_{L}(0,1)\left(1-\lambda\left(C_{L}\right)\right) \lambda\left(C_{R}\right)+\xi_{L}(0,0)\left(1-\lambda\left(C_{L}\right)\right)\left(1-\lambda\left(C_{R}\right)\right) .
$$

This includes all those who have seen the advertisement of Party $L$ 's candidate; a fraction $\xi_{L}(0,1)$ of those who have seen only the advertisement of Party $R$ 's candidate; and a fraction $\xi_{L}(0,0)$ of those who have seen neither candidate's advertisement. The fraction of right-leaning moderates voting for Party L's candidate is

$$
\delta_{R}\left(C_{L}, C_{R}\right)=\xi_{R}(1,0)\left(1-\lambda\left(C_{R}\right)\right) \lambda\left(C_{L}\right)+\xi_{R}(0,0)\left(1-\lambda\left(C_{R}\right)\right)\left(1-\lambda\left(C_{L}\right)\right) .
$$

It includes a fraction $\xi_{R}(1,0)$ of those who have seen only the advertisement of Party $L$ 's candidate; and a fraction $\xi_{R}(0,0)$ of those who have seen neither candidate's advertisement.

The fraction of moderates voting for Party $L$ 's candidate is $\mu \delta_{L}+(1-\mu) \delta_{R}$. Given the assumption that partisans are equally split between rightists and leftists, Party L's candidate will win if this fraction exceeds $1 / 2$ or, equivalently, if $\mu \geq\left(1 / 2-\delta_{R}\right) /\left(\delta_{L}-\delta_{R}\right)$. This implies that 
the probability that Party L's candidate wins is $\pi\left(C_{L}, C_{R}\right)$, where the probability of winning function $\pi$ is defined as follows: $\pi\left(C_{L}, C_{R}\right)=0$ if $\left(1 / 2-\delta_{R}\right) /\left(\delta_{L}-\delta_{R}\right) \geq 1, \pi\left(C_{L}, C_{R}\right)=1$ if $\left(1 / 2-\delta_{R}\right) /\left(\delta_{L}-\delta_{R}\right) \leq 0$, and

$$
\pi\left(C_{L}, C_{R}\right)=1-\frac{1 / 2-\delta_{R}\left(C_{L}, C_{R}\right)}{\delta_{L}\left(C_{L}, C_{R}\right)-\delta_{R}\left(C_{L}, C_{R}\right)}=\frac{\delta_{L}\left(C_{L}, C_{R}\right)-1 / 2}{\delta_{L}\left(C_{L}, C_{R}\right)-\delta_{R}\left(C_{L}, C_{R}\right)}
$$

otherwise.

If only Party $L$ 's candidate is a moderate, he wins with probability $\pi\left(C_{L}, 0\right)$. Similarly, if only Party $R$ 's candidate is a moderate, the probability that Party $L$ 's candidate wins is $\pi\left(0, C_{R}\right)$. If both candidates are partisans, then no contributions are given and Party L's candidate wins with probability $\pi(0,0)$.

\subsection{Interest group contributions}

The contribution levels provided to moderate candidates by the two interest groups must be mutual best responses given voter behavior. The interest groups do not observe the type of the opposing party's candidate when making their contributions and seek to maximize the expected payoff of their representative member. Thus, the leftist interest group's contribution $C_{L}$ maximizes

$$
\chi_{R} \pi\left(C, C_{R}\right) \beta(1-2 x)+\left(1-\chi_{R}\right) \pi(C, 0) \beta(1-x-d)-2 C /(1-\gamma) \theta
$$

where $\chi_{R}$ is the probability it assigns to Party $R$ selecting a moderate. Similarly, the rightist interest group's contribution $C_{R}$ maximizes

$$
\chi_{L}\left(1-\pi\left(C_{L}, C\right)\right) \beta(1-2 x)+\left(1-\chi_{L}\right)(1-\pi(0, C)) \beta(1-x-d)-2 C /(1-\gamma) \theta
$$

where $\chi_{L}$ is the probability it assigns to Party $L$ selecting a moderate.

\subsection{Candidate selection}

Parties choose the type of candidate to run anticipating interest group contributions and voter behavior. They seek to maximize the payoff of their representative member, who is assumed not 
to belong to the interest group. ${ }^{13}$ Let $\tau_{K} \in\{M, P\}$ denote the type of Party $K$ 's candidate and let $U_{K}\left(\tau_{L}, \tau_{R}\right)$ denote the expected payoff of a partisan of type $K$ if the types of candidates selected are $\left(\tau_{L}, \tau_{R}\right) \cdot{ }^{14}$ We let $\sigma_{K}$ denote the probability that Party $K$ selects a moderate and assume that $\left(\sigma_{L}, \sigma_{R}\right)$ are mutual best responses for the two parties.

\subsection{Political equilibrium}

A political equilibrium consists of (i) candidate selection strategies for the two parties $\left(\sigma_{L}, \sigma_{R}\right)$ describing the probability that each party selects a moderate; (ii) contribution levels for the two interest groups $\left(C_{L}, C_{R}\right)$ describing the contributions they make to moderate candidates; (iii) interest group beliefs $\left(\chi_{R}, \chi_{L}\right)$ describing the probabilities they assign to the opposing party having chosen a moderate; (iv) voting behavior functions $\left(\xi_{L}\left(I_{L}, I_{R}\right), \xi_{R}\left(I_{L}, I_{R}\right)\right)$ describing moderates' voting behavior as a function of the information they have received in the campaign; and (v) voter belief functions $\left(\rho_{L}\left(I_{L}, I_{R}\right), \rho_{R}\left(I_{L}, I_{R}\right)\right)$ describing moderates' beliefs concerning candidate types as a function of the information they have. Candidate selection strategies must be mutual best responses given subsequent interest group and voter behavior. Interest groups' contribution strategies must be mutual best responses given subsequent voter behavior and their beliefs. Voter behavior must be consistent with their beliefs. Interest groups beliefs must be consistent with parties strategies and voters' beliefs must be consistent with the strategies of parties and interest groups.

The analysis will focus on political equilibria that are symmetric in the sense that the two parties choose moderates with the same probability (i.e., $\sigma_{L}=\sigma_{R}=\sigma$ ); interest groups contribute the same amount to their parties' moderate candidates (i.e., $C_{L}=C_{R}=C$ ); moderates vote in

\footnotetext{
13 If the representative party member also belonged to the interest group, parties would need to take into account the cost of the contributions their candidates would receive.

14 Thus,

$U_{L}(M, M)=\pi\left(C_{L}, C_{R}\right) \beta(1-2 x)-\beta(1-x-d)$, and

$U_{R}(M, M)=\left(1-\pi\left(C_{L}, C_{R}\right)\right) \beta(1-2 x)-\beta(1-x-d)$, and so on.
} 
the same way (i.e., $\xi_{L}(0,0)=1-\xi_{R}(0,0)$ and $\xi_{L}(0,1)=1-\xi_{R}(1,0)$ ); and moderates' beliefs about the two candidates are symmetric (i.e., $\rho_{L}(0,0)=\rho_{R}(0,0)$ and $\rho_{L}(0,1)=\rho_{R}(1,0)$ ). In such an equilibrium, moderates who have observed neither candidate's advertisement are loyal to their party's candidate; i.e., $\xi_{L}(0,0)=1-\xi_{R}(0,0)=1$. Thus, letting $\xi=\xi_{R}(1,0)$ and $\rho=\rho_{L}(0,1)$, a symmetric political equilibrium may be described by the vector $(\sigma, C, \xi, \rho)$. There is no need to include interest group beliefs in the description of equilibrium, since these are simply $\sigma$.

The variable $\xi$ represents the fraction of right-leaning (left-leaning) moderates who vote for Party $L$ 's candidate (Party $R$ 's candidate) when they have only observed an advertisement from this candidate. It measures the effectiveness of campaign advertising in inducing moderate voters to switch from their natural allegiances. When $\xi$ is high, voters are easily swayed and when $\xi$ is low, campaign advertising is ineffective. Together with the two interest groups' contributions, $\xi$ determines the fractions of left and right-wing moderates voting for their party's candidate. We recognize this dependence by writing the probability of winning function as $\pi\left(C_{L}, C_{R} ; \xi\right)$.

Voters' loyalty is determined by $\rho$ - their beliefs concerning the probability that their party's candidate is moderate when they have only seen an advertisement from the opposing candidate. Assuming that $\sigma>0$ and $C>0, \rho$ is tied down by Bayes Rule. However, if $\sigma=0$, then the event of observing one candidate's advertisement does not arise along the equilibrium path and $\rho$ is not tied down. Thus, there exist symmetric equilibria in which $\sigma=0$ and $\rho$ is large enough to make $\xi$ so small as to make campaign advertising ineffective. Since it seems unreasonable to suppose that $\rho$ is anything other than 0 when both parties are selecting partisans with probability one, I focus only on symmetric equilibria which have the property that $\sigma=0$ implies that $\rho=0 .{ }^{15}$ Henceforth, a symmetric equilibrium is understood to be an equilibrium satisfying this additional requirement.

\footnotetext{
${ }^{15}$ For a more technical defense of this focus, note that equilibria in which $\sigma=0$ and $\rho>0$ do not satisfy the requirement that there exist a sequence $\left(\sigma_{n}, C_{n}\right)_{n=1}^{\infty}$ such that $\left(\sigma_{n}, C_{n}\right) \in(0,1) \times(0, \infty)$ and $\lim _{n \rightarrow \infty}\left(\sigma_{n}, C_{n}\right)=$ $(\sigma, C)$ with the property that $\lim _{n \rightarrow \infty} \rho_{n}=\rho$ where $\rho_{n}$ are the beliefs implied by Bayes Rule given $\left(\sigma_{n}, C_{n}\right)$. Thus, such equilibria are not sequential equilibria (Kreps and Wilson (1982)).
} 


\subsection{Welfare}

For welfare analysis, the expected payoffs of the various types of citizens in a symmetric political equilibrium need to be calculated. With probability $\sigma^{2}$ both parties select a moderate and with probability $2 \sigma(1-\sigma)$ only one party selects a moderate. In the former case, both candidates spend $C$ on their campaigns and, in the latter, the moderate candidate spends $C$. Thus, expected campaign spending is $E=\left[2 \sigma(1-\sigma) C+\sigma^{2} 2 C\right]=2 C \sigma$. Moreover, when there is only one moderate, he wins with probability $\pi(C, 0 ; \xi)$ and hence the probability that a moderate is elected is $\sigma^{2}+2 \sigma(1-\sigma) \pi(C, 0 ; \xi)$.

Consider a leftist who is not an interest group member. Given symmetry, the elected candidate is equally likely to be from either party. The expected payoff of the leftist if the elected candidate is a partisan is therefore $-\beta(1-2 d) / 2$. If, on the other hand, the elected candidate is a moderate the payoff of the leftist is $-\beta[(x-d) / 2+(1-x-d) / 2]$ which again equals $-\beta(1-2 d) / 2$. Thus, the leftist does not benefit from the elected candidate being more likely to be a moderate: the gain from a lower chance of getting a rightist is just offset by the loss from an reduced chance of getting a leftist. ${ }^{16}$ Similar remarks apply to rightists who are not interest group members. Interest group members finance campaign spending and hence obtain expected payoffs $-\beta(1-2 d) / 2-E /(1-\gamma) \theta$.

Now consider a representative moderate. From an ex-ante perspective, he will be left-leaning with probability $\mu$ and right-leaning with probability $1-\mu$ where $\mu$ is the realization of a uniformly distributed random variable. Computing his payoff is complicated by the correlation between which party's candidate wins and the likelihood that he is left or right-leaning. To illustrate, suppose that both parties have selected moderates. If $\mu<1 / 2$ then the majority of moderates are right-leaning and Party $R$ 's candidate will win. Accordingly, the representative moderate's expected payoff is $-\mu \beta(1-2 x)$. If $\mu>1 / 2$ then Party $L$ 's candidate wins and the representative

\footnotetext{
16 This result does depend on the assumption that citizens have distance preferences. If instead, we assumed that citizens had preferences of the form $v\left(\left|i-i^{\prime}\right|\right)$ where $v$ is decreasing and strictly concave, then the leftist would benefit from the elected candidate being more likely to be moderate.
} 
moderate's expected payoff is $-(1-\mu) \beta(1-2 x)$. Taking expectations over $\mu$, the expected welfare of a moderate when both parties select moderates is therefore

$$
-\int_{0}^{\frac{1}{2}} \mu \beta(1-2 x) d \mu-\int_{\frac{1}{2}}^{1}(1-\mu) \beta(1-2 x) d \mu=-\frac{\beta(1-2 x)}{4} .
$$

The key point is that states in which the representative moderate is more likely to be left-leaning are states in which Party L's candidate will win. Indeed, ensuring that this is the case is the key function of elections in this model.

Pursuing this logic for the cases in which both parties select partisans and only one party selects a moderate, the expected payoff of a representative moderates can be shown to equal $W_{M}(\sigma, \pi(C, 0 ; \xi))$ where,

$$
\begin{aligned}
W_{M}(\sigma, \pi)= & -\sigma^{2} \frac{\beta(1-2 x)}{4}-(1-\sigma)^{2}\left(\frac{\beta(1-x-d)}{4}+\frac{3 \beta(x-d)}{4}\right) \\
& -2 \sigma(1-\sigma)\left\{\pi\left[\frac{\pi}{2} \beta(1-2 x)\right]+(1-\pi)\left[\frac{1-\pi}{2} \beta(1-x-d)+\frac{1+\pi}{2} \beta(x-d)\right]\right\} .
\end{aligned}
$$

The second and third terms are, respectively, the expected payoffs when parties select partisans and only one party selects a moderate. A detailed derivation of them can be found in the Appendix.

Aggregate expected welfare for the community is given by:

$$
W(\sigma, \pi(C, 0 ; \xi), E)=\gamma W_{M}(\sigma, \pi(C, 0 ; \xi))-(1-\gamma) \beta(1-2 d) / 2-E
$$

Aggregate welfare depends on three key variables: the probability that parties select moderates, the probability that a moderate defeats a partisan, and expected campaign spending.

\section{Characterization of political equilibrium}

In this section, the unique symmetric equilibrium is characterized. We first understand how much the interest groups will contribute to candidates, taking as given the effectiveness of campaign advertising. Then, taking into account this contribution behavior, we examine how parties choose candidates. Finally, we solve for voters' behavior. 
The following assumption on the parameter values is maintained throughout the analysis.

Assumption 1: $\frac{\beta \theta(1-\gamma)(1-x-d)}{4 \alpha}>1$.

This assumption ensures that equilibrium involves positive levels of campaign contributions. Without it, interest groups do not find it worthwhile to contribute to moderate candidates. Accordingly, parties experience no electoral advantage from selecting moderates and equilibrium involves both parties choosing partisans.

\subsection{Campaign contributions}

Given $\sigma$ and $\xi$, a contribution level $C$ is a mutual best response for the two interest groups if and only if

$$
C=\arg \max _{C_{L} \geq 0}\left\{\sigma \pi\left(C_{L}, C ; \xi\right) \beta(1-2 x)+(1-\sigma) \pi\left(C_{L}, 0 ; \xi\right) \beta(1-x-d)-2 C_{L} /(1-\gamma) \theta\right\}
$$

Our assumptions imply that $\pi\left(C_{L}, C ; \xi\right)$ is a concave function of $C_{L}$ when $\delta_{L}\left(C_{L}, C\right)<\frac{1}{2}<$ $\delta_{R}\left(C_{L}, C\right)$. Moreover, $\frac{\partial \pi(C, C ; \xi)}{\partial C_{L}}$ is a decreasing function of $C .{ }^{17}$ These facts allow us to establish the following result, the proof of which may be found in the Appendix.

Lemma 1 For all $(\sigma, \xi) \in[0,1]^{2}$ there exists a unique contribution level that is a mutual best response for the two interest groups.

We denote this unique level by $\widehat{C}(\sigma, \xi)$. Obviously, if $(\sigma, C, \xi, \rho)$ is an equilibrium then it must be the case that $C=\widehat{C}(\sigma, \xi)$. On the range of the parameter space in which contributions are such that a moderate candidate defeats a partisan with a probability between $\frac{1}{2}$ and 1 (i.e., $\left.\pi(\widehat{C}, 0 ; \xi) \in\left(\frac{1}{2}, 1\right)\right)$, interest groups give more if there is a higher probability that their party's candidate will face a partisan, so $\widehat{C}(\sigma, \xi)$ is decreasing in $\sigma$. Moreover, interest groups give more when campaign advertising is more effective so that $\widehat{C}$ is increasing in $\xi .^{18}$ In addition, when an

\footnotetext{
17 The assumptions that make the probability of winning function well-behaved are those concerning the distribution of $\mu$ and the advertising technology.

18 This property does not necessarily hold when $\pi(\widehat{C}, 0 ; \xi)=1$, since reducing $\xi$ will necessitate an increase in contributions to maintain certain victory.
} 
interest group believes the other side will select a partisan for sure $(\sigma=0)$ and when campaign advertising is maximally effective $(\xi=1)$, then it gives an amount sufficient to ensure the victory of its party's candidate with probability one. This and the previous claims are established in the Appendix.

\subsection{Parties' choice of candidates}

When choosing the type of candidate to select, parties take as given voters' behavior and the contributions moderate candidates will attract from the interest groups. This behavior determines the probability that each type of candidate will win. In a symmetric equilibrium, if both parties choose the same type of candidate, each party's candidate wins with equal probability. Thus, for each Party $K, U_{K}(\tau, \tau)=-\beta(1-2 d) / 2$ for $\tau \in\{M, P\}$. If one party chooses a moderate and the other a partisan, the probability that the moderate wins is $\pi(C, 0 ; \xi)$. Thus, the party running the moderate obtains the payoff

$$
U_{L}(M, P)=U_{R}(P, M)=\pi(C, 0 ; \xi) \beta(1-x-d)-\beta(1-2 d),
$$

and the party running the partisan obtains

$$
U_{L}(P, M)=U_{R}(M, P)=-\pi(C, 0 ; \xi) \beta(1-x-d) .
$$

Irrespective of the type of candidate the opposing party runs, the gain from running a moderate as opposed to a partisan is

$$
U_{L}\left(M, \tau_{R}\right)-U_{L}\left(P, \tau_{R}\right)=U_{R}\left(\tau_{L}, M\right)-U_{R}\left(\tau_{L}, P\right)=\pi(C, 0 ; \xi) \beta(1-x-d)-\frac{\beta(1-2 d)}{2} .
$$

This "gain" is increasing in the probability that a moderate defeats a partisan. It is also negative when $\pi(C, 0 ; \xi)=\frac{1}{2}$ and positive when $\pi(C, 0 ; \xi)=1$. Thus, letting

$$
\pi^{*}=\frac{1-2 d}{2(1-x-d)}
$$


running a moderate is a dominant strategy for each party if $\pi(C, 0 ; \xi)$ exceeds $\pi^{*}$, while running a partisan is a dominant strategy for each party if $\pi(C, 0 ; \xi)$ is less than $\pi^{*}$. If $\pi(C, 0 ; \xi)$ equals $\pi^{*}$, then each party is indifferent between running a partisan and a moderate. It follows that, if $(\sigma, C, \xi, \rho)$ is a symmetric equilibrium, then $\sigma=0$ if $\pi(C, 0 ; \xi)$ is less than $\pi^{*}$ and $\sigma=1$ if $\pi(C, 0 ; \xi)$ exceeds $\pi^{*}$.

\subsection{Voter behavior}

The next task is to understand, for given $\sigma$, how voters respond to advertising. Recall first that the effectiveness of campaign advertising $\xi$ is given by:

$$
\xi=\xi_{R}(1,0)=1-H\left(v_{R}(R ; 1,0)-v_{R}(L ; 1,0)\right)
$$

It is the case that $v_{R}(R ; 1,0)=-(1-\rho) \beta(x-d)$ and $v_{R}(L ; 1,0)=-\beta(1-2 x)$ and hence the above equation implies that $\xi$ is related to voters' beliefs about the probability an unadvertised candidate is moderate $\rho$ in the following way:

$$
\xi=1-H(\rho \beta(x-d)-\beta(3 x-1-d))
$$

Letting $\bar{\rho}=[\beta(3 x-1-d)+\bar{\varepsilon}] / \beta(x-d)$ and $\underline{\rho}=[\beta(3 x-1-d)-\bar{\varepsilon}] / \beta(x-d)$, our earlier assumptions on the size of $\bar{\varepsilon}$ imply that campaign advertising is completely ineffective $(\xi=0)$ when $\rho \geq \bar{\rho}$ and maximally effective $(\xi=1)$ when $\rho \leq \underline{\rho}$.

Voters' beliefs about unadvertised candidates must be consistent with the strategies of parties and interest groups. Thus, assuming that $\sigma>0$ and $C>0$, Bayes Rule implies that voters' beliefs are given by

$$
\rho=\frac{\sigma[1-\lambda(C)]}{\sigma[1-\lambda(C)]+(1-\sigma)}
$$

Moreover, by assumption, if $\sigma=0$, then $\rho=0$. We can now establish that, in equilibrium, parties must be indifferent between running a moderate and a partisan.

Lemma 2 Let $(\sigma, C, \xi, \rho)$ be a symmetric political equilibrium. Then, $\pi(C, 0 ; \xi)=\pi^{*}$. 
To understand this result, note that if moderates defeated partisans with a probability greater than $\pi^{*}$ then both parties would choose moderates. But then campaign advertising would be completely ineffective because moderate voters would know that their party's candidate was moderate and hence would never switch their votes to the opposing party's candidate. If advertising was ineffective, interest groups would make no contributions and hence moderates would have no advantage against partisans - a contradiction. If instead moderates defeated partisans with a probability less than $\pi^{*}$, both parties would choose partisans. But then campaign advertising would be fully effective which implies that moderate candidates would receive sufficient contributions to defeat partisans with probability one - a contradiction.

Now define $\bar{\sigma}$ to be the highest probability with which parties may select moderates that is consistent with parties receiving sufficient contributions to be indifferent. Formally, $\bar{\sigma}=\max \{\sigma \in$ $\left.[0,1]: \pi(\widehat{C}(\sigma, 1), 0 ; 1) \geq \pi^{*}\right\}$. If $\bar{\sigma}<1$ and $\sigma>\bar{\sigma}$, then interest groups will not contribute sufficiently to moderate candidates to ensure that they defeat partisans with a probability $\pi^{*}$ even when campaign spending is maximally effective. Thus, by Lemma 2, such a $\sigma$ cannot be part of a symmetric equilibrium. In the Appendix, it is demonstrated that

$$
\bar{\sigma}=\min \left\{1, \frac{\left[\left(2 \pi^{*}-1\right)^{2}+1\right][\beta(1-\gamma) \theta(1-x-d)-4 \alpha]}{\beta(1-\gamma) \theta\left[(1-x-d)\left(2 \pi^{*}-1\right)^{2}+x-d\right]}\right\}
$$

Observe that $\bar{\sigma}$ will be close to 0 when the parameters are such that Assumption 1 only just holds.

Next, for all $\sigma \in[0, \bar{\sigma}]$ let $\widehat{\xi}(\sigma)$ solve the equation $\pi(\widehat{C}(\sigma, \xi), 0 ; \xi)=\pi^{*}$. Intuitively, $\widehat{\xi}(\sigma)$ is the level of advertising effectiveness that would induce interest groups to contribute an amount sufficient to make a moderate candidate win with probability $\pi^{*}$ against a partisan when they expect the opposing party to choose a moderate with probability $\sigma$. Lemma 2 implies that if $(\sigma, C, \xi, \rho)$ is a symmetric equilibrium, it must be the case $\xi=\widehat{\xi}(\sigma)$, which determines voters' behavior as a function of $\sigma$.

Several properties of $\widehat{\xi}(\sigma)$ should be noted. First, since $\pi(\widehat{C}(0,1), 0 ; 1)=1, \widehat{\xi}(0)$ must be less 
than 1. Second, the function $\widehat{\xi}(\cdot)$ is increasing on $[0, \bar{\sigma}]$ because the higher the probability the opposing party selects a moderate the lower the incentive to contribute. Finally, if $\bar{\sigma}$ is less than 1 , it is the case that $\widehat{\xi}(\bar{\sigma})=1$.

\subsection{Political equilibrium}

At this point, we have established that if $(\sigma, C, \xi, \rho)$ is a symmetric political equilibrium then the effectiveness of campaign advertising is $\widehat{\xi}(\sigma)$, the level of campaign contributions is $\widehat{C}(\sigma, \widehat{\xi}(\sigma))$, and $\sigma$ must belong to the interval $[0, \bar{\sigma}]$. Moreover, the equilibrium belief that an unadvertised candidate is moderate must be such as to generate the voting behavior $\widehat{\xi}(\sigma)$ and, since $\sigma>0,{ }^{19}$ $\sigma$ must be related to $C$ and $\rho$ via Bayes Rule. More formally, for any given value of $\xi$, let $\widehat{\rho}(\xi)$ be the set of voter beliefs that are consistent with such behavior. ${ }^{20}$ Then, it must be the case that $\rho \in \widehat{\rho}(\widehat{\xi}(\sigma))$ and that

$$
\sigma=\frac{\rho}{\rho+(1-\rho)(1-\lambda(\widehat{C}(\sigma, \widehat{\xi}(\sigma))))} .
$$

Conversely, if there exists $\sigma \in(0, \bar{\sigma}]$ with the property that there exists $\rho^{\prime} \in \widehat{\rho}(\widehat{\xi}(\sigma))$ such that the above equation holds, then $\left(\sigma, \widehat{C}(\sigma, \widehat{\xi}(\sigma)), \widehat{\xi}(\sigma), \rho^{\prime}\right)$ is a symmetric equilibrium. These observations allow us to establish the following result:

Proposition 1 There exists a unique symmetric political equilibrium. In this equilibrium, parties select moderates with positive probability. Moderate candidates receive campaign contributions from interest groups that allow them to defeat partisans with a probability between $\frac{1}{2}$ and 1 .

In equilibrium, contributions finance the provision of information about candidates to voters and increase the probability that the candidate who would be majority preferred if voters had perfect information wins. They therefore help to produce outcomes closer to those that would arise under perfect information. Moreover, the fact that candidates may receive contributions

\footnotetext{
${ }^{19}$ We can rule out the possibility that $\sigma=0$, since that implies (by assumption) that $\rho=0$ and this belief cannot generate the voting behavior $\widehat{\xi}(0)$ since $\widehat{\xi}(0)$ is less than 1 .

${ }^{20}$ Formally, $\widehat{\rho}(\xi)=\{\rho \in[0,1]: \xi=1-H(\rho \beta(x-d)-\beta(3 x-1-d))\}$.
} 
provides parties with an incentive to select candidates with characteristics that the majority of voters want. Thus the model provides a framework in which campaign contributions play the role that they are commonly understood to play.

Depending on the parameter values, the equilibrium is either interior or boundary. In an interior equilibrium, parties select moderates with a probability $\sigma$ less than $\bar{\sigma}$ and campaign advertising is less than fully effective $(\xi<1)$. In such an equilibrium, the level of campaign spending exceeds $\alpha\left(2 \pi^{*}-1\right)$. In a boundary equilibrium, parties select moderates with probability $\bar{\sigma}$, campaign advertising is maximally effective $(\xi=1)$, and campaign spending equals $\alpha\left(2 \pi^{*}-1\right)$. The equilibrium will be a boundary equilibrium if $\bar{\sigma}<\underline{\rho} 2 \pi^{*} /\left[\underline{\rho} 2 \pi^{*}+1-\underline{\rho}\right]^{21}$ Since $\underline{\rho}>0$, equilibrium will be of the boundary variety when the parameters are such that Assumption 1 is only just satisfied.

\subsection{Is there too much campaign spending in equilibrium?}

In popular debate concerning campaign finance reform in the United States, the question of whether there is too much or too little money spent on campaigns is often raised. Our model suggests one way of thinking about this rather nebulous question. Let $\left(\sigma^{*}, C^{*}, \xi^{*}, \rho^{*}\right)$ be the symmetric equilibrium. Given the behavior of parties and voters as described by $\sigma^{*}$ and $\xi^{*}$, the contribution level that maximizes aggregate welfare is $C^{o}=\arg \max W\left(\sigma^{*}, \pi\left(C, 0, \xi^{*}\right), 2 \sigma^{*} C\right)$. If $C^{*}$ is less than $C^{o}$, there is a natural sense in which contributions are underprovided. For if interest groups were to give a little more, then, holding constant the behavior of parties and voters, aggregate welfare would increase. It should be stressed that underprovision according to this definition has no obvious implications for policy. For any policy effort to increase interest groups' contributions will alter the behavior of parties and voters. Nonetheless, the definition

\footnotetext{
21 This follows from the proof of Proposition 1. Using the notation introduced in the proof, we know that $\widehat{\xi}(\bar{\sigma})=1, f(0)=\underline{\rho}$ and $\widehat{C}(\underline{\sigma}, 0)=\alpha\left(2 \pi^{*}-1\right)$ if $\bar{\sigma}<1$. It is now straightforward to verify that $\varphi(\bar{\sigma}) \geq 0$ if and only if the above inequality is satisfied.
} 
facilitates a clear understanding of the (first order) divergences between the private and social incentives to contribute.

The optimal level (assuming it is positive) satisfies the first order condition:

$$
\gamma \frac{\partial W_{M}\left(\sigma^{*}, \pi\left(C^{o}, 0 ; \xi^{*}\right)\right)}{\partial \pi} \frac{\partial \pi\left(C^{o}, 0 ; \xi^{*}\right)}{\partial C_{L}}=2 \sigma^{*}
$$

Computing the derivative $\partial W_{M} / \partial \pi$, we obtain

$$
2 \sigma^{*}\left(1-\sigma^{*}\right) \frac{\partial \pi\left(C^{o}, 0 ; \xi^{*}\right)}{\partial C_{L}} \gamma\left\{\beta(1-x-d)-2 \pi\left(C^{o}, 0 ; \xi^{*}\right) \beta(1-2 x)\right\}=2 \sigma^{*}
$$

The left hand side represents the social marginal benefit of contributions which is that they raise the probability that a moderate defeats a partisan. This raises the expected aggregate welfare of moderates, assuming that $\frac{1-x-d}{2(1-2 x)}>\pi(\cdot) .^{22} \quad$ The right hand side represents the social marginal cost which is the increase in expected expenditures. The equilibrium level of contributions, on the other hand, satisfies the first order condition:

$$
\sigma^{*} \frac{\partial \pi\left(C^{*}, C^{*} ; \xi^{*}\right)}{\partial C_{L}} \beta(1-2 x)+\left(1-\sigma^{*}\right) \frac{\partial \pi\left(C^{*}, 0 ; \xi^{*}\right)}{\partial C_{L}} \beta(1-x-d)=\frac{2}{(1-\gamma) \theta}
$$

The left hand side represents the private marginal benefit of contributions to an interest group member and the right hand side represents the private marginal cost.

These expressions reveal two sources of differences between the private and social incentives to contribute. First, from a social perspective campaign contributions are only useful when moderates are running against partisans. Thus, if the probability of such races is low because both parties are very likely to pick moderates then the social marginal benefit of contributions will be small or

\footnotetext{
22 It is interesting to note that if $x<(1+d) / 3$ then $\frac{1-x-d}{2(1-2 x)}<1$ so it is possible that $\pi$ could be so high that raising the probability that a moderate defeats a partisan may actually be harmful to moderates. To understand this, note that there are three effects of raising $\pi$ on the expected payoff of a representative moderate. The first is to increase the likelihood that when a moderate runs against a partisan, the moderate wins. This effect is positive, because moderates receive a higher expected payoff when a moderate is elected. The second effect is to decrease the size of the expected payoff arising when a moderate wins. This occurs because raising $\pi$ increases the likelihood that the representative moderate will end up with a moderate candidate of the opposing ideology. The third effect is to increase the expected payoff arising when a partisan wins. Raising $\pi$ increases the likelihood that the representative moderate will be on the same side as the partisan. The overall effect is negative when the second effect dominates the first and third.
} 
zero. By contrast, interest groups still have an incentive to contribute even if they know that the opposing party is very likely to select a moderate. This reflects the purely distributional incentive that they would prefer the moderate on their side of the ideological fence to win. Second, even when moderate versus partisan races are likely, the private incentives to contribute do not match up with the social incentives. The former reflect the fraction of interest group members and the gains to these citizens from having a moderate from their own side as opposed to a partisan from the other. The latter reflect the fraction of moderates in the population and the gain to them of raising the probability that a moderate defeats a partisan.

These divergences mean that contributions can be over or under-provided according to our definition. ${ }^{23}$ Overprovision arises when the probability that parties select moderates is high. In this case, campaign contributions have no direct social benefit and are purely wasteful rent seeking in the sense of Tullock (1980). ${ }^{24}$ Underprovision arises when parties select partisans with a significant probability and the fraction of interest group members $(1-\gamma) \theta / 2$ is small relative to the fraction of moderates $\gamma$. In this case, campaign contributions have a significant social benefit but this is not internalized by interest group members who care only about their own (purely redistributive) benefit.

As noted in Section 2, the possibility of both over and underprovision of advertising also arises in markets with differentiated products. The fact that the benefits firms obtain from advertising come partially at the expense of their competitors corresponds to the purely redistributive nature of the benefits interest groups get from financing political advertising. Moreover, the fact that firms do not take into account the surplus their advertising creates for consumers is similar to

\footnotetext{
23 Specific numerical examples are available from the author on request.

24 See Congelton (1986) for an interesting discussion of political advertising as rent-seeking. He observes that political advertising has rent-seeking aspects since it involves competition for a distributional gain. However, in contrast to standard rent-seeking, it has the spillover benefit of providing valuable information to voters. Thus, it is not clear that it produces rent-seeking losses of the usual form. Our analysis provides a concrete way of thinking about the informational benefits and sheds light on the circumstances under which they will be large or small relative to the rent-seeking losses.
} 
interest groups not taking into account the benefits of their activities to moderate voters.

\section{The impact of contribution limits}

We now consider a policy that puts an upper limit on the contributions that interest groups can make. We investigate the impact that such a limit will have on equilibrium and when limits will enhance aggregate welfare. The limit will be denoted by $L$. Obviously, to have any impact, $L$ must be less than the laissez-faire level of campaign contributions and this is assumed in the sequel.

\subsection{Equilibrium with contribution limits}

If the contribution limit is sufficiently small so that $\pi(L, 0 ; 1)<\pi^{*}$, then, no matter how effective campaign advertising is, both parties will select partisans. The probability of a moderate defeating a partisan is simply insufficient to induce parties to select one. In such circumstances, equilibrium involves both parties selecting partisans. Noting that the condition that $\pi(L, 0 ; 1)<\pi^{*}$ is equivalent to $L<\alpha\left[2 \pi^{*}-1\right]$, yields:

Proposition 2 Suppose that the contribution limit $L$ is less than $\alpha\left[2 \pi^{*}-1\right]$. Then, there exists a unique symmetric political equilibrium. In this equilibrium, parties select partisans and expected campaign spending is zero.

A contribution limit less than this critical value effectively eliminates all campaign spending. Even though moderate candidates would attract contributions, parties do not select moderates because the electoral advantages from so doing are not sufficient to compensate for the compromise in ideology. Hence no campaign spending arises in equilibrium. It follows from this proposition that if the laissez-faire equilibrium is a boundary equilibrium so that $C=\alpha\left[2 \pi^{*}-1\right]$, then even a very small contribution limit will completely eliminate campaign spending.

If the contribution limit is such that $\pi(L, 0 ; 1) \geq \pi^{*}$, equilibrium has a similar structure to the laissez-faire. In particular, as the following result shows, the probability that a moderate defeats 
a partisan must be the same as in the laissez-faire.

Lemma 3 Suppose that the contribution limit $L$ is at least as big as $\alpha\left[2 \pi^{*}-1\right]$ and let $(\sigma, C, \xi, \rho)$ be a symmetric political equilibrium. Then, $C=L$ and $\xi$ solves the equation $\pi(L, 0 ; \xi)=\pi^{*}$.

Thus, if the contribution limit is large enough, equilibrium still has the property that parties are indifferent between selecting moderate and partisan candidates. The equation $\pi(L, 0 ; \xi)=$ $\pi^{*}$ has a unique solution $\widetilde{\xi}(L)=(L+\alpha)\left(2 \pi^{*}-1\right) / 2 L \pi^{*}$. Note that $\widetilde{\xi}(L)$ is decreasing, implying that stricter contribution limits increase the effectiveness of campaign advertising. Campaign advertising must be more effective if the probability of a partisan defeating a moderate candidate remains the same.

The equilibrium belief that an unadvertised candidate is moderate must be such as to generate the voting behavior $\widetilde{\xi}(L)$, which implies that $\rho \in \widehat{\rho}(\widetilde{\xi}(L))$. If $\sigma>0$, then $\sigma$ is related to $\rho$ by the familiar equation

$$
\sigma=\frac{\rho}{\rho+(1-\rho)(1-\lambda(L))} .
$$

If $\sigma=0$, then $\rho=0$ and hence $\widetilde{\xi}(L)=1$. This requires that $L=\alpha\left(2 \pi^{*}-1\right)$. With this background, we are now able to establish:

Proposition 3 If the contribution limit $L$ is bigger than $\alpha\left[2 \pi^{*}-1\right]$ there exists a unique symmetric political equilibrium, while if the limit $L$ is exactly $\alpha\left[2 \pi^{*}-1\right]$ there are multiple symmetric equilibria. In either case, imposing the limit does not change the probability that a moderate candidate defeats a partisan. However, the limit reduces both the probability that parties select moderates and expected campaign spending. The smaller the limit, the larger are these reductions.

To sum up, contribution limits that are at least as big as $\alpha\left[2 \pi^{*}-1\right]$ do not impact the probability a moderate defeats a partisan. Rather, they reduce the probability that parties select moderates. Intuitively, because limits reduce campaign advertising but do not impact the probability a moderate defeats a partisan, the effectiveness of campaign advertising must increase. The 
only way this can happen is that voters believe that unadvertised candidates are less likely to be moderates. This in turn requires that parties select moderates with a lower probability.

\subsection{Contribution limits and welfare}

The distributive consequences of contribution limits are clear from Propositions 2 and 3. Moderates are made worse off because the probability of parties selecting moderates is reduced. ${ }^{25}$ Leftists and rightists who do not belong to interest groups are unaffected, while interest group members are actually made better off because they spend less of their resources on campaign contributions. Thus imposing contribution limits redistributes from moderates to interest group members.

Less clear is the aggregate welfare impact of contribution limits. Are the gains to interest group members more than offset by the losses to moderates? To investigate this, let $\left(\sigma^{*}, C^{*}, \xi^{*}, \rho^{*}\right)$ denote the laissez-faire equilibrium and let $\left(\sigma^{*}(L), C^{*}(L), \xi^{*}(L), \rho^{*}(L)\right)$ denote the equilibrium with contribution limit $L .{ }^{26}$ Similarly, let $E^{*}$ denote expected campaign spending in the laissezfaire and $E^{*}(L)$ spending with limit $L$. We are interested in comparing $W\left(\sigma^{*}, \pi\left(C^{*}, 0, \xi^{*}\right), E^{*}\right)$ and $W\left(\sigma^{*}(L), \pi\left(C^{*}(L), 0, \xi^{*}(L)\right), E^{*}(L)\right)$. Matters are simplified by noting that if $L$ is bigger than $\alpha\left[2 \pi^{*}-1\right]$ imposing the limit has no impact on the probability that moderates defeat partisans. Moreover, if $L$ is smaller than $\alpha\left[2 \pi^{*}-1\right]$ then, parties never select moderates and hence the probability that a moderate defeats a partisan is irrelevant. Thus, for analytical purposes, we may assume that the limit leaves unchanged the probability a moderate defeats a partisan and only impacts the probability that parties select moderates and expected campaign spending. This permits a diagrammatic analysis.

Such an analysis is presented in Figure 1 under the assumption that the laissez-faire is an interior equilibrium. The horizontal axis measures expected campaign spending and the vertical

\footnotetext{
${ }^{25}$ It is readily verified that $W_{M}(\sigma, \pi(C, 0 ; \xi))$ is increasing in $\sigma$.

${ }^{26}$ If $L=\alpha\left(2 \pi^{*}-1\right)$, let $\left(\sigma^{*}(L), C^{*}(L), \xi^{*}(L), \rho^{*}(L)\right)$ be any one of the multiple possible equilibria.
} 
axis measures the probability that parties select a moderate. The family of indifference curves represent social preferences over different $(E, \sigma)$ combinations. These indifference curves are upward sloping, convex, and parallel to each other. ${ }^{27}$ Since the social ideal would be to have parties select moderates for sure and no campaign spending, moving to the left yields higher levels of welfare.

The laissez-faire outcome is represented by the point $\left(E^{*}, \sigma^{*}\right)$. Introducing a limit lowers both the probability that parties select moderates and expected campaign spending. The curve joining the points $\left(E^{*}, \sigma^{*}\right)$ and $(\widehat{E}, \widehat{\sigma})$ is obtained by successively reducing the limit from $C^{*}$ to $\alpha\left(2 \pi^{*}-1\right)$ and tracing out the points $\left(E^{*}(L), \sigma^{*}(L)\right)$. When the limit reaches $\alpha\left[2 \pi^{*}-1\right]$, there are multiple $(E, \sigma)$ combinations that are possible equilibria. The highest of these is the point $(\widehat{E}, \widehat{\sigma})$ and the lowest is $(0,0) \cdot{ }^{28}$ The remaining possible equilibrium combinations lie on a straight line connecting $(\widehat{E}, \widehat{\sigma})$ and $(0,0) \cdot{ }^{29}$ The slope of this line can be shown to be larger than the slope of the curve joining the points $\left(E^{*}, \sigma^{*}\right)$ and $(\widehat{E}, \widehat{\sigma})$ at the point $(\widehat{E}, \widehat{\sigma})$. Reducing the limit below $\alpha\left(2 \pi^{*}-1\right)$ yields the point $(0,0)$. The curve joining $(0,0)$ to $\left(E^{*}, \sigma^{*}\right)$ therefore represents the locus of possibilities that society might achieve with contribution limits.

To determine whether introducing a particular limit $L$ increases or decreases social welfare, we need to locate the point on the possibility locus that the limit generates and see if this point lies on a higher social indifference curve than the laissez-faire outcome. In Figure 1, social indifference curves are sufficiently flat so that any contribution limit puts society on a lower social indifference curve and hence reduces aggregate welfare. Obviously, this is not the only possibility.

Figure 1 assumes that the laissez-faire equilibrium is interior. If it is a boundary equilibrium,

\footnotetext{
27 The indifference curves are implicitly defined by the equality $W\left(\sigma, \pi^{*}, E\right)=K$ for some constant $K$. The indifference curves have slope $\frac{d \sigma}{d E}=\frac{1}{\gamma \partial W_{M}\left(\sigma, \pi^{*}\right) / \partial \sigma}>0$ and second derivative $\frac{d^{2} \sigma}{d E^{2}}=\frac{-\gamma \partial^{2} W_{M}\left(\sigma, \pi^{*}\right) / \partial \sigma^{2}}{\left(\gamma \partial W_{M}\left(\sigma, \pi^{*}\right) / \partial \sigma\right)^{3}}>0$.

28 It can be shown that $(\widehat{E}, \widehat{\sigma})=\left(\frac{\underline{\rho} \alpha\left(4 \pi^{*}\right)\left(2 \pi^{*}-1\right)}{\underline{\rho}\left(2 \pi^{*}-1\right)+1}, \frac{\underline{\rho} 2 \pi^{*}}{\underline{\rho}\left(2 \pi^{*}-1\right)+1}\right)$.

29 This is established in the proof of Proposition 4 below.
} 
the laissez-faire outcome $\left(E^{*}, \sigma^{*}\right)$ must lie on the curve connecting $(\widehat{E}, \widehat{\sigma})$ and $(0,0)$. In this case, society's possibility locus just consists of the points $\left(E^{*}, \sigma^{*}\right)$ and $(0,0)$ and the question of the desirability of contribution limits is very simple.

We can now use this diagrammatic apparatus to understand the following proposition:

Proposition 4: (i) If $\beta>\frac{4 \alpha[\theta(x-d)+1-2 x]}{\theta(1-2 x)(1-x-d)}$ there exists $\bar{\gamma}<1-\frac{4 \alpha}{\beta \theta(1-x-d)}$ such that for all $\gamma \in\left(\bar{\gamma}, 1-\frac{4 \alpha}{\beta \theta(1-x-d)}\right)$, imposing any contribution limit would reduce aggregate welfare. (ii) There exists $\underline{\gamma}>0$, such that for all $\gamma \in(0, \underline{\gamma})$, there exists a welfare enhancing contribution limit. Part (i) provides a condition on $\beta$ under which imposing contribution limits is always welfare reducing if the fraction of moderates is sufficiently large. Part (ii) says that if moderates make up a sufficiently small fraction of the population, then there will exist contribution limits that would improve welfare. The latter result is easy to understand once one realizes that the social indifference curves become steeper and steeper as the fraction of moderates goes to zero. To understand part (i), note that as we increase the fraction of moderates, the size of the interest groups decreases and hence the generosity of contributions decreases. Eventually, the laissez-faire equilibrium becomes a boundary equilibrium. Accordingly, the laissez-faire outcome must lie on the line connecting $(\widehat{E}, \widehat{\sigma})$ and $(0,0)$ and any contribution limit must move society to the point $(0,0)$. Under the stated condition on $\beta$, the point $(\widehat{E}, \widehat{\sigma})$ must lie on a higher social indifference curve than $(0,0)$ for sufficiently large values of $\gamma$ in the admissible range. ${ }^{30}$ By the convexity of the indifference curves, any point $(E, \sigma)$ lying on the line is also socially preferred to $(0,0)$ and hence the result.

Proposition 4 suggests the possibility of a stronger result which says that, under the condition on $\beta$, contribution limits are desirable if and only if the fraction of moderates is below some critical level. This is indeed the case if, for all $\gamma$, the curve joining the points $\left(E^{*}, \sigma^{*}\right)$ and $(\widehat{E}, \widehat{\sigma})$ in Figure

30 The admissible range consists of those values consistent with Assumption 1 holding. 
1 is concave. ${ }^{31}$ While this is the case when $\varepsilon$ is uniformly distributed (i.e., $H(\varepsilon)=\frac{\varepsilon+\bar{\varepsilon}}{2 \bar{\varepsilon}}$ ), it would not appear to be true in general. Since, in principle, it is possible for the result not to hold in the presence of non-concavities, Proposition 4 appears the best general result available.

\section{Conclusion}

This paper has developed a model of political competition with campaign contributions and informative advertising. In equilibrium, contributions finance the provision of information about candidates to voters and increase the probability that the candidate who would be majority preferred were voters perfectly information wins. Contributions therefore help to produce more informed choices. Moreover, the existence of campaign contributions provides parties with an incentive to select candidates with characteristics that the majority of voters want. Thus the model provides a framework in which campaign contributions play the role they are commonly understood to play in reality.

The model facilitates analysis of the often raised question of whether there is too much or too little spending on campaigns. Taking as given parties' candidate selection strategies and the effectiveness of campaign advertising, one can ask whether aggregate welfare would be higher if interest groups provided more or less money to candidates. In general, equilibrium spending levels

\footnotetext{
31 To see this, let $W\left(\sigma, \pi^{*}, E ; \gamma\right)$ denote the aggregate welfare function and $C^{*}(\gamma)$ the laissez-faire level of campaign spending when the fraction of moderates is $\gamma$. Similarly, let $\left(E^{*}(\gamma), \sigma^{*}(\gamma)\right)$ denote the probability that parties select moderates and expected campaign spending in the laissez-faire and $\left(E^{*}(L ; \gamma), \sigma^{*}(L ; \gamma)\right)$ these variables with limit $L$. We need to show that if for some $\widehat{\gamma}$ there exists $\widehat{L}<C^{*}(\widehat{\gamma})$ such that $W\left(\sigma^{*}(\widehat{\gamma}), \pi^{*}, E^{*}(\widehat{\gamma}) ; \widehat{\gamma}\right)$ is smaller than $W\left(\sigma^{*}(\widehat{L} ; \widehat{\gamma}), \pi^{*}, E^{*}(\widehat{L} ; \widehat{\gamma}) ; \widehat{\gamma}\right)$ then, if $\gamma^{\prime}<\widehat{\gamma}$, there exists some $L^{\prime}<C^{*}\left(\gamma^{\prime}\right)$ such that $W\left(\sigma^{*}\left(\gamma^{\prime}\right), \pi^{*}, E^{*}\left(\gamma^{\prime}\right) ; \gamma^{\prime}\right)$ is smaller than $W\left(\sigma^{*}\left(L^{\prime} ; \gamma^{\prime}\right), \pi^{*}, E^{*}\left(L^{\prime} ; \gamma^{\prime}\right) ; \gamma^{\prime}\right)$. Interest groups are bigger when $\gamma$ is lower which leads to more contributions and hence a higher probability of electing moderates and higher campaign expenditures. Thus, $\left(E^{*}\left(\gamma^{\prime}\right), \sigma^{*}\left(\gamma^{\prime}\right)\right)$ lies to the northeast of the point $\left(E^{*}(\widehat{\gamma}), \sigma^{*}(\widehat{\gamma})\right)$. Note also that the equilibrium with contribution limits is independent of $\gamma$ provided that $L<C^{*}(\gamma)$. This means that $(\widehat{E}, \widehat{\sigma})$ is independent of $\gamma$, and that the curve connecting $\left(E^{*}\left(\gamma^{\prime}\right), \sigma^{*}\left(\gamma^{\prime}\right)\right)$ to $(\widehat{E}, \widehat{\sigma})$ coincides with that connecting $\left(E^{*}(\widehat{\gamma}), \sigma^{*}(\widehat{\gamma})\right)$ to $(\widehat{E}, \widehat{\sigma})$ for limits $L$ less than or equal to $C^{*}(\widehat{\gamma})$. Since $W\left(\sigma^{*}(\widehat{\gamma}), \pi^{*}, E^{*}(\widehat{\gamma}) ; \widehat{\gamma}\right)$ is smaller than $W\left(\sigma^{*}(\widehat{L} ; \widehat{\gamma}), \pi^{*}, E^{*}(\widehat{L} ; \widehat{\gamma}) ; \widehat{\gamma}\right)$, it follows that $W\left(\sigma^{*}\left(C^{*}(\widehat{\gamma}) ; \gamma^{\prime}\right), \pi^{*}, E^{*}\left(C^{*}(\widehat{\gamma}) ; \gamma^{\prime}\right) ; \widehat{\gamma}\right)$ is smaller than $W\left(\sigma^{*}\left(\widehat{L} ; \gamma^{\prime}\right), \pi^{*}, E^{*}\left(\widehat{L} ; \gamma^{\prime}\right) ; \widehat{\gamma}\right)$. If the curve connecting $\left(E^{*}\left(\gamma^{\prime}\right), \sigma^{*}\left(\gamma^{\prime}\right)\right)$ to $(\widehat{E}, \widehat{\sigma})$ is concave it is easy to see diagrammatically that this must imply that $W\left(\sigma^{*}\left(\gamma^{\prime}\right), \pi^{*}, E^{*}\left(\gamma^{\prime}\right) ; \widehat{\gamma}\right)$ is smaller than $W\left(\sigma^{*}\left(\widehat{L} ; \gamma^{\prime}\right), \pi^{*}, E^{*}\left(\widehat{L} ; \gamma^{\prime}\right) ; \widehat{\gamma}\right)$. Since the social indifference curves are flatter when the fraction of moderates is $\gamma^{\prime}$, this implies that $W\left(\sigma^{*}\left(\gamma^{\prime}\right), \pi^{*}, E^{*}\left(\gamma^{\prime}\right) ; \gamma^{\prime}\right)$ is smaller than $W\left(\sigma^{*}\left(L^{*} ; \gamma^{\prime}\right), \pi^{*}, E^{*}\left(L^{*} ; \gamma^{\prime}\right) ; \gamma^{\prime}\right)$.
} 
may be too low or too high because of the divergences between the private incentives of interest groups to contribute and the social benefits of such contributions. Overprovision arises when parties are highly likely to select moderates, in which case campaign contributions have no social benefit. Underprovision arises when parties are more likely to select partisans and interest group membership is small relative to the number of moderates. In this case, campaign contributions have a social benefit that dwarfs the purely distributional benefits accruing to interest group members.

While the question of too much or too little spending is an interesting one, the model makes clear that any change in the regulation of campaign contributions is likely to impact not only contribution levels but also parties' candidate selection strategies and the effectiveness of campaign advertising. Contribution limits were shown to reduce the likelihood that parties select moderates and raise the effectiveness of campaign spending. The impact of limits on citizens' welfare is redistributive. Moderate voters are made worse off. Partisans who do not belong to interest groups are unaffected because the increase in the likelihood of electing a partisan of their own ideology is offset by the increase in the probability of electing a partisan of the opposing ideology. Partisans who are interest group members are actually made better off because they spend less of their resources on campaign contributions. The question of whether the gains to interest group members offset the losses to moderate voters turns on the fraction of the latter in the population. Contribution limits are always desirable from an aggregate viewpoint when only a small fraction of the population are moderates, while limits will tend to be harmful when moderates dominate.

This paper represents only a small step in developing an understanding of campaign finance and its regulation. Nonetheless, the framework could readily be extended to shed further light. One useful extension would be to introduce asymmetries in the power of the interest groups supporting the two parties. This could be done by assuming either that a larger fraction of partisans were interest group members on one side or, perhaps more realistically, that partisans on one side had 
greater wealth and hence a higher willingness to contribute. It would be interesting to understand how such asymmetries might influence candidate selection and bias election outcomes. In this context, the familiar argument that limits "level the political playing field" could be scrutinized.

Much of the demand for campaign finance reform is motivated by the belief that giving by big donors is service induced. One could introduce service induced contributions into the framework by assuming that candidates could approach interest groups and offer to provide policy favors in exchange for contributions. Then, seeing advertising would lead voters to increase their estimates that a candidate would enact special interest legislation when elected and hence dampen the effectiveness of campaign spending. Contribution limits might then impact the amount of policy favors provided by reducing the incentives for candidates to solicit contributions. This in turn may have interesting implications for the effectiveness of campaign advertising.

Finally, there are many interesting questions concerning the allocation of campaign resources across different uses (see also Schultz (2001)). One could, for example, analyze the welfare implications of negative advertising by allowing candidates to provide information to voters about their opponents' records. In the model of this paper, this would provide a reason for contributing to partisan candidates. Alternatively one could introduce another dimension of difference between candidates (such as competence) and study candidates' decisions as to which characteristic to campaign on. 


\section{References}

Austen-Smith, David, [1987], "Interest Groups, Campaign Contributions and Probabilistic Voting," Public Choice, 54, 123-139.

Austen-Smith, David, [1997], "Interest Groups: Money, Information, and Influence," in Perspectives on Public Choice, Dennis Mueller (ed), Cambridge University Press.

Baron, David, [1994], "Electoral Competition with Informed and Uniformed Voters," American Political Science Review, 88, 33-47.

Bazaraa, Mokhtar and C.M. Shetty, [1979], Nonlinear Programming: Theory and Algorithms, John Wiley \& Sons.

Besley, Timothy and Stephen Coate, [1997], "An Economic Model of Representative Democracy," Quarterly Journal of Economics, 112(1), 85-114.

Besley, Timothy and Stephen Coate, [2000], "Issue Unbundling via Citizens' Initiatives," NBER Working Paper \#8036.

Besley, Timothy and Stephen Coate, [2001], "Elected versus Appointed Regulators: Theory and Evidence," typescript.

Congleton, Roger, [1986], "Rent-seeking Aspects of Political Advertising," Public Choice, 49, 249-63.

Grossman, Gene and Elhanan Helpman, [1996], "Electoral Competition and Special Interest Politics," Review of Economic Studies, 63(2), 265-286.

Grossman, Gene and Elhanan Helpman, [2001], Special Interest Politics, Cambridge, MIT Press.

Grossman, Gene and Carl Shapiro, [1984], "Informative Advertising with Differentiated Products," Review of Economic Studies, 51, 63-81.

Kreps, David and Robert Wilson, [1982], "Sequential Equilibrium," Econometrica, 50, 863894 .

Lindbeck, Assar and Jorgen Weibull, [1987], "Balanced Budget Redistribution as the Outcome of Political Competition," Public Choice, 52, 273-297.

Milgrom, Paul and John Roberts, [1986], "Price and Advertising Signals of Product Quality," Journal of Political Economy, 94, 796-821.

Morton, Rebecca and Charles Cameron, [1992], "Elections and the Theory of Campaign Contributions: A Survey and Critical Analysis," Economics and Politics, 4, 79-108.

Nelson, Phillip, [1976], "Political Information," Journal of Law and Economics, 19, 315-336.

Ortuno-Ortin, Ignacio and Christian Schultz, [2000], "Public Funding for Political Parties," CESifo Working Paper No. 368. 
Osborne, Martin J. and Al Slivinski, [1996], "A Model of Political Competition with Citizen Candidates", Quarterly Journal of Economics, 111(1), 65-96.

Persson, Torsten and Guido Tabellini, [2000], Political Economics: Explaining Economic Policy, Cambridge, MIT Press.

Potters, Jan, Randolph Sloof and Frans van Winden, [1997], "Campaign Expenditures, Contributions, and Direct Endorsements: The Strategic Use of Information and Money to Influence Voter Behavior," European Journal of Political Economy, 13, 1-31.

Prat, Andrea, [1999], "Campaign Advertising and Voter Welfare," mimeo, London School of Economics.

Prat, Andrea, [2000], "Campaign Spending with Office-Seeking Politicians, Rational Voters, and Multiple Lobbies," mimeo, London School of Economics (forthcoming Journal of Economic Theory).

Schultz, Christian, [2001], "Strategic Campaigns and Special Interest Politics," mimeo, University of Copenhagen.

Tullock, Gordon, [1980], "Efficient Rent-Seeking," in Towards a Theory of the Rent-Seeking Society, James Buchanan, Robert Tollison and Gordon Tullock (eds), Texas A\&M University Press. 


\section{Appendix}

Derivation of the Expected Payoff of the Representative Moderate: It was shown in the text that when both parties select moderates, a representative moderate's expected payoff is $-\beta(1-2 x) / 4$. Suppose now that both parties select partisans. When $\mu<1 / 2$, Party $R$ 's candidate wins. The representative moderate's expected payoff is therefore $-\mu \beta(1-x-d)-(1-$ $\mu) \beta(x-d)$. If $\mu>1 / 2$, Party $L$ 's candidate wins and the representative moderate's expected payoff is $-\mu \beta(x-d)-(1-\mu) \beta(1-x-d)$. Taking expectations over the realization of $\mu$, the expected payoff of the representative moderate is:

$$
-\int_{\frac{1}{2}}^{1}[\mu \beta(x-d)+(1-\mu) \beta(1-x-d)] d \mu-\int_{0}^{\frac{1}{2}}[\mu \beta(1-x-d)+(1-\mu) \beta(x-d)] d \mu .
$$

This equals

$$
-\left(\frac{\beta(1-x-d)}{4}+\frac{3 \beta(x-d)}{4}\right)
$$

Next, suppose that one party has selected a moderate and the other a partisan. For concreteness, suppose that Party $L$ has selected a moderate. Party $R$ 's candidate will win if $\mu$ is less than $\frac{1 / 2-\delta_{R}(C, 0)}{\delta_{L}(C, 0)-\delta_{R}(C, 0)}$. The representative moderate's expected payoff is then $-\mu \beta(1-x-d)-$ $(1-\mu) \beta(x-d)$. If $\mu$ exceeds $\frac{1 / 2-\delta_{R}(C, 0)}{\delta_{L}(C, 0)-\delta_{R}(C, 0)}$, Party $L$ 's candidate will win and the representative moderate's expected payoff is $-(1-\mu) \beta(1-2 x)$. Thus, the expected payoff of the representative moderate is:

$$
-\int_{\frac{1 / 2-\delta_{R}}{\delta_{L}-\delta_{R}}}^{1}(1-\mu) \beta(1-2 x) d \mu-\int_{0}^{\frac{1 / 2-\delta_{R}}{\delta_{L}-\delta_{R}}}[\mu \beta(1-x-d)+(1-\mu) \beta(x-d)] d \mu .
$$

Using the fact that $\pi(C, 0 ; \xi)=1-\frac{1 / 2-\delta_{R}(C, 0)}{\delta_{L}(C, 0)-\delta_{R}(C, 0)}$, we can write this as

$-\pi(C, 0 ; \xi)\left[\frac{\pi(C, 0 ; \xi)}{2} \beta(1-2 x)\right]-(1-\pi(C, 0 ; \xi))\left[\frac{1-\pi(C, 0 ; \xi)}{2} \beta(1-x-d)+\frac{1+\pi(C, 0 ; \xi)}{2} \beta(x-d)\right]$.

Noting that the probabilities that (i) both parties select moderates, (ii) both parties select partisans, and (iii) only one party selects a moderate are, respectively, $\sigma^{2},(1-\sigma)^{2}$, and $2 \sigma(1-\sigma)$ yields the welfare expression given in the text. 
Proof of Lemma 1: Let $(\sigma, \xi) \in[0,1]^{2}$ be given. For all $\left(C_{L}, C\right) \in \Re_{+}^{2}$ let

$$
f\left(C_{L}, C\right)=\sigma \pi\left(C_{L}, C ; \xi\right) \beta(1-2 x)+(1-\sigma) \pi\left(C_{L}, 0 ; \xi\right) \beta(1-x-d)-2 C_{L} / \theta(1-\gamma) .
$$

Then, we must show that there exists a unique $C$ such that $C=\arg \max _{C_{L} \geq 0} f\left(C_{L}, C\right)$.

The first step is to compute the probability of winning function. Using the functional form for $\lambda$, we obtain

$$
\delta_{L}\left(C_{L}, C_{R}\right)=\frac{C_{L}\left(\alpha+C_{R}\right)+\alpha\left((1-\xi) C_{R}+\alpha\right)}{\left(\alpha+C_{L}\right)\left(\alpha+C_{R}\right)},
$$

and

$$
\delta_{R}\left(C_{L}, C_{R}\right)=\frac{\xi \alpha C_{L}}{\left(\alpha+C_{L}\right)\left(\alpha+C_{R}\right)} .
$$

Note that $\delta_{L}\left(C_{L}, C_{R}\right)<\frac{1}{2}$ if and only if $C_{L}<\frac{\alpha\left[C_{R}(2 \xi-1)-\alpha\right]}{\alpha+C_{R}}$ and that $\delta_{R}\left(C_{L}, C_{R}\right)>\frac{1}{2}$ if and only if $C_{L}\left[\alpha(2 \xi-1)-C_{R}\right]>\alpha\left(\alpha+C_{R}\right)$. Thus, we have that $\pi\left(C_{L}, C_{R} ; \xi\right)=0$ if $C_{L}<\frac{\alpha\left[C_{R}(2 \xi-1)-\alpha\right]}{\alpha+C_{R}}$; $\pi\left(C_{L}, C_{R} ; \xi\right)=1$ if $C_{L}\left[\alpha(2 \xi-1)-C_{R}\right]>\alpha\left(\alpha+C_{R}\right)$ and

$$
\pi\left(C_{L}, C_{R} ; \xi\right)=\frac{C_{L}\left(\alpha+C_{R}\right) / 2+\alpha(1 / 2-\xi) C_{R}+\alpha^{2} / 2}{C_{L}\left(\alpha(1-\xi)+C_{R}\right)+\alpha(1-\xi) C_{R}+\alpha^{2}}
$$

otherwise. Over the range in which $\delta_{R}<\frac{1}{2}<\delta_{L}$ the effect of a small increase in interest group $L$ 's contribution on the winning probability is

$$
\frac{\partial \pi}{\partial C_{L}}=\frac{\alpha \xi\left\{C_{R} \alpha 2(1-\xi)+C_{R}^{2}+\alpha^{2}\right\}}{2\left\{C_{L}\left(\alpha(1-\xi)+C_{R}\right)+\alpha(1-\xi) C_{R}+\alpha^{2}\right\}^{2}}
$$

which is positive as long as $\xi>0$. In addition, note that $\frac{\partial^{2} \pi}{\partial C_{L}^{2}}<0$ so that $\pi\left(\cdot, C_{R} ; \xi\right)$ is a concave function over this range.

It will be useful to define the following functions:

$h\left(C_{L}, C\right)=\sigma \frac{C_{L}(\alpha+C) / 2+\alpha(1 / 2-\xi) C+\alpha^{2} / 2}{C_{L}(\alpha(1-\xi)+C)+\alpha(1-\xi) C+\alpha^{2}} \beta(1-2 x)+(1-\sigma) \frac{C_{L}+\alpha}{2\left(C_{L}(1-\xi)+\alpha\right)} \beta(1-x-d)-2 C_{L} / \theta(1-\gamma)$,

and

$g\left(C_{L}, C\right)=\sigma \frac{C_{L}(\alpha+C) / 2+\alpha(1 / 2-\xi) C+\alpha^{2} / 2}{C_{L}(\alpha(1-\xi)+C)+\alpha(1-\xi) C+\alpha^{2}} \beta(1-2 x)+(1-\sigma) \beta(1-x-d)-2 C_{L} / \theta(1-\gamma)$. 
Note that, for all $C, h(\cdot, C)$ and $g(\cdot, C)$ are concave and continuously differentiable functions. Moreover, their derivatives are such that $\frac{\partial h\left(C_{L}, C\right)}{\partial C_{L}}>\frac{\partial g\left(C_{L}, C\right)}{\partial C_{L}}$.

Suppose first that $\xi \leq \frac{1}{2}$. Then, for all $\left(C_{L}, C_{R}\right)$ it is clear that it must be the case that $\delta_{R}\left(C_{L}, C_{R}\right)<\frac{1}{2}<\delta_{L}\left(C_{L}, C_{R}\right)$. This implies that $f\left(C_{L}, C\right)=h\left(C_{L}, C\right)$. It follows from the properties of the function $h(\cdot, C)$ that $C=\arg \max _{C_{L} \geq 0} f\left(C_{L}, C\right)$ if and only if $\frac{\partial h(C, C)}{\partial C_{L}} \leq 0$ with equality if $C>0$. Thus, we need to show that there exists a unique $C \geq 0$ with this property. Differentiating, we obtain

$$
\frac{\partial h(C, C)}{\partial C_{L}}=\frac{\sigma \xi \alpha \beta(1-2 x)}{2\left(2(1-\xi) \alpha C+C^{2}+\alpha^{2}\right)}+\frac{(1-\sigma) \xi \alpha \beta(1-x-d)}{2((1-\xi) C+\alpha)^{2}}-\frac{2}{\theta(1-\gamma)}
$$

Viewed as a function of $C, \frac{\partial h(C, C)}{\partial C_{L}}$ is continuous, decreasing, and negative for $C$ sufficiently large. Thus, if $\frac{\partial h(0,0)}{\partial C_{L}} \leq 0, C=0$ has the desired property. Otherwise, the $C$ at which $\frac{\partial h(C, C)}{\partial C_{L}}=0$ is the unique $C$ with the desired property.

Now suppose that $\xi>\frac{1}{2}$. Then, letting $\widehat{f}\left(C_{L}, C\right)=\min \left\{h\left(C_{L}, C\right), g\left(C_{L}, C\right)\right\}$, we have that:

$$
\begin{aligned}
f\left(C_{L}, C\right) & =-2 C_{L} / \theta(1-\gamma) \text { if } C_{L}<\max \left\{0, \frac{\alpha[C(2 \xi-1)-\alpha]}{\alpha+C}\right\}, \\
f\left(C_{L}, C\right) & =\sigma \beta(1-2 x)+(1-\sigma) \beta(1-x-d)-2 C_{L} / \theta(1-\gamma) \text { if } C_{L}[\alpha(2 \xi-1)-C]>\alpha(\alpha+C), \\
f\left(C_{L}, C\right) & =\widehat{f}\left(C_{L}, C\right) \text { otherwise. }
\end{aligned}
$$

We now have:

Claim: $C=\arg \max _{C_{L} \geq 0} f\left(C_{L}, C\right)$ if and only if $C=\arg \max _{C_{L} \geq 0} \widehat{f}\left(C_{L}, C\right)$ and $\widehat{f}(C, C) \geq 0$.

Proof of Claim: Let $C=\arg \max _{C_{L} \geq 0} f\left(C_{L}, C\right)$. If $C \leq \frac{\alpha}{2 \xi-1}$ then $f\left(C_{L}, C\right)=\widehat{f}\left(C_{L}, C\right)$ for all $C_{L} \geq 0$ and hence it is immediate that $C=\arg \max _{C_{L} \geq 0} \widehat{f}\left(C_{L}, C\right)$. Moreover, $\widehat{f}(C, C) \geq$ $\widehat{f}(0, C) \geq 0$.

If $C>\frac{\alpha}{1-2 \xi}$ then since it must be the case that $C>\frac{\alpha[C(2 \xi-1)-\alpha]}{\alpha+C}$ and $C<\frac{\alpha(\alpha+C)}{\alpha(2 \xi-1)-C}$ we know that $f(C, C)=\widehat{f}(C, C)$. Let $\widetilde{C}_{L}=\arg \max _{C_{L} \geq 0} \widehat{f}\left(C_{L}, C\right)$. It is the case that $\widehat{f}\left(C_{L}, C\right)=h\left(C_{L}, C\right)$ if $C_{L} \leq \frac{\alpha}{2 \xi-1}$ and $\widehat{f}\left(C_{L}, C\right)=g\left(C_{L}, C\right)$ if $C_{L}>\frac{\alpha}{2 \xi-1}$. Since $\frac{\partial g\left(C_{L}, C\right)}{\partial C_{L}}$ is negative for sufficiently 
large $C_{L}, \widetilde{C}_{L}$ exists. If $\widetilde{C}_{L} \neq C$, then either $\widetilde{C}_{L}<\frac{\alpha[C(2 \xi-1)-\alpha]}{\alpha+C}$ or $\widetilde{C}_{L}>\frac{\alpha(\alpha+C)}{\alpha(2 \xi-1)-C}$. In the former case, we must have that $\widehat{f}(C, C)<\widehat{f}\left(\widetilde{C}_{L}, C\right)=h\left(\widetilde{C}_{L}, C\right) \leq f\left(\widetilde{C}_{L}, C\right)$ which is a contradiction. In the latter case, we must have that $\widehat{f}\left(\frac{\alpha(\alpha+C)}{\alpha(2 \xi-1)-C}, C\right)>\widehat{f}\left(C_{L}, C\right)$ for all $C_{L}<\frac{\alpha(\alpha+C)}{\alpha(2 \xi-1)-C}$. This in turn implies that $f\left(\frac{\alpha(\alpha+C)}{\alpha(2 \xi-1)-C}, C\right)>f\left(C_{L}, C\right)$ for all $C_{L} \neq \frac{\alpha(\alpha+C)}{\alpha(2 \xi-1)-C}$ which implies that $C=\frac{\alpha(\alpha+C)}{\alpha(2 \xi-1)-C}$ - a contradiction. Finally, note that $\widehat{f}(C, C)=f(C, C) \geq f(0, C) \geq 0$.

Now let $C=\arg \max _{C_{L} \geq 0} \widehat{f}\left(C_{L}, C\right)$ and $\widehat{f}(C, C) \geq 0$. If $C \leq \frac{\alpha}{2 \xi-1}$ then $f\left(C_{L}, C\right)=\widehat{f}\left(C_{L}, C\right)$ for all $C_{L} \geq 0$ and hence it is immediate that $C=\arg \max _{C_{L} \geq 0} f\left(C_{L}, C\right)$. If $C>\frac{\alpha}{2 \xi-1}$ then for all $C_{L}<\frac{\alpha[C(2 \xi-1)-\alpha]}{\alpha+C}$, we have that $f\left(C_{L}, C\right) \leq 0 \leq \widehat{f}(C, C)$. Moreover, for all $C_{L}>\frac{\alpha(\alpha+C)}{\alpha(2 \xi-1)-C}$ we have that $f\left(C_{L}, C\right) \leq \widehat{f}\left(\frac{\alpha(\alpha+C)}{\alpha(2 \xi-1)-C}, C\right) \leq \widehat{f}(C, C)$. Thus, $C=\arg \max _{C_{L} \geq 0} f\left(C_{L}, C\right)$. QED

Notice that $\widehat{f}(\cdot, C)$ as the minimum of two concave functions is itself concave. It follows that $C=\arg \max _{C_{L} \geq 0} \widehat{f}\left(C_{L}, C\right)$ if and only if $\widehat{f}$ has a subgradient $\phi$ at $C$ such that $\phi\left(C_{L}-C\right) \leq 0$ for all $C_{L} \geq 0$ (see, for example, Theorem 3.4.3 of Bazaraa and Shetty (1979)). Thus, by the Claim, it suffices to establish that there exists a unique $C$ at which there is a subgradient with the desired property and that this $C$ also satisfies the inequality $\widehat{f}(C, C) \geq 0$.

Note first that $\widehat{f}\left(C_{L}, C\right)=h\left(C_{L}, C\right)$ if $C_{L} \leq \frac{\alpha}{2 \xi-1}$ and $\widehat{f}\left(C_{L}, C\right)=g\left(C_{L}, C\right)$ if $C_{L}>\frac{\alpha}{2 \xi-1}$. It follows that the subgradient of $\widehat{f}(\cdot, C)$ at any $C_{L}$ is the derivative $\frac{\partial h\left(C_{L}, C\right)}{\partial C_{L}}$ if $C_{L}<\frac{\alpha}{2 \xi-1}$ and the derivative $\frac{\partial g\left(C_{L}, C\right)}{\partial C_{L}}$ if $C_{L}>\frac{\alpha}{2 \xi-1}$. If $C_{L}=\frac{\alpha}{2 \xi-1}$, then $\phi$ is a subgradient of $f$ if and only if $\phi \in\left[\frac{\partial g\left(\frac{\alpha}{2 \xi-1}, C\right)}{\partial C_{L}}, \frac{\partial h\left(\frac{\alpha}{2 \xi-1}, C\right)}{\partial C_{L}}\right]$.

If $\frac{\partial h(0,0)}{\partial C_{L}} \leq 0$, then $\frac{\partial h(0,0)}{\partial C_{L}} C_{L} \leq 0$ for all $C_{L} \geq 0$ and hence $C=0$ has the desired property. Moreover, it is the unique $C$ with the property for if $C^{\prime}>0$ then, since $\frac{\partial h(C, C)}{\partial C_{L}}$ is decreasing and $\frac{\partial h(C, C)}{\partial C_{L}}>\frac{\partial g(C, C)}{\partial C_{L}}$ for all $C$, the subgradient of $\widehat{f}\left(\cdot, C^{\prime}\right)$ at $C^{\prime}$ is negative which implies that $C^{\prime}$ cannot have the desired property. Finally, note that $\widehat{f}(0,0)=h(0,0)>0$.

If $\frac{\partial h(0,0)}{\partial C_{L}}>0$ and $\frac{\partial h\left(\frac{\alpha}{2 \xi-1}, \frac{\alpha}{2 \xi-1}\right)}{\partial C_{L}}<0$, then there exists a unique $C \in\left(0, \frac{\alpha}{2 \xi-1}\right)$ such that $\frac{\partial h(C, C)}{\partial C_{L}}=0$ and this is the unique $C$ with the desired property. It satisfies the inequality since 
$\widehat{f}(C, C)=h(C, C)>h(0, C) \geq 0$.

If $\frac{\partial h\left(\frac{\alpha}{2 \xi-1}, \frac{\alpha}{2 \xi-1}\right)}{\partial C_{L}} \geq 0$ and $\frac{\partial g\left(\frac{\alpha}{2 \xi-1}, \frac{\alpha}{2 \xi-1}\right)}{\partial C_{L}} \leq 0$, then there exists a subgradient of $\widehat{f}\left(\cdot, \frac{\alpha}{2 \xi-1}\right)$ at $C_{L}=$ $\frac{\alpha}{2 \xi-1}$ which satisfies $\phi=0$ and hence $C=\frac{\alpha}{2 \xi-1}$ has the desired property. This is also the unique $C$ with the property. Moreover, it satisfies the inequality since $\widehat{f}\left(\frac{\alpha}{2 \xi-1}, \frac{\alpha}{2 \xi-1}\right)=h\left(\frac{\alpha}{2 \xi-1}, \frac{\alpha}{2 \xi-1}\right)>$ $h\left(0, \frac{\alpha}{2 \xi-1}\right)=0$.

If $\frac{\partial g\left(\frac{\alpha}{2 \xi-1}, \frac{\alpha}{2 \xi-1}\right)}{\partial C_{L}}>0$, then since $\frac{\partial g(C, C)}{\partial C_{L}}$ is continuous, decreasing and negative for $C$ sufficiently large, there exists a unique $C$ such that $\frac{\partial g(C, C)}{\partial C_{L}}=0$ and this is the unique $C$ with the desired property. To show that it satisfies the inequality, we need to establish that:

$$
g(C, C)=\frac{\sigma}{2} \beta(1-2 x)+(1-\sigma) \beta(1-x-d)-2 C / \theta(1-\gamma) \geq 0
$$

Note that

$$
\frac{\partial g(C, C)}{\partial C_{L}}=\frac{\sigma \xi \alpha \beta(1-2 x)}{2\left(2(1-\xi) \alpha C+C^{2}+\alpha^{2}\right)}-\frac{2}{\theta(1-\gamma)}=0
$$

which implies that

$$
\beta(1-2 x)=\frac{4\left(2(1-\xi) \alpha C+C^{2}+\alpha^{2}\right)}{(1-\gamma) \theta \sigma \xi \alpha}
$$

Thus,

$$
g(C, C)>\frac{2}{(1-\gamma) \theta \xi \alpha}\left[\left(2(1-\xi) \alpha C+C^{2}+\alpha^{2}\right)-\xi \alpha C\right]>0
$$

where the last inequality follows from the fact that $C>\frac{\alpha}{2 \xi-1}>\alpha$. QED

Proof of Properties of $\widehat{C}(\sigma, \xi)$ : First we show that on the range of the parameter space in which $\pi(\widehat{C}(\sigma, \xi), 0,0) \in\left(\frac{1}{2}, 1\right), \widehat{C}$ is decreasing in $\sigma$ and increasing in $\xi$. From the proof of Lemma 1 , we know that over this range $\widehat{C}(\sigma, \xi)$ is implicitly defined by the equation $\frac{\partial h(\widehat{C}, \widehat{C})}{\partial C_{L}}=0$. Since $\frac{\partial h(C, C)}{\partial C_{L}}$ is decreasing in $C$, it suffices to show that $\frac{\partial h(C, C)}{\partial C_{L}}$ is decreasing in $\sigma$ and increasing in $\xi$. We leave this to the reader.

Next we show that $\widehat{C}(0,1)=\alpha$, which implies that $\pi(\widehat{C}(0,1), 0 ; 1)=1$. When $\sigma=0$ and $\xi=1$ 
then for all $C$

$$
\frac{\partial h(C, C)}{\partial C_{L}}=\frac{\beta(1-x-d)}{2 \alpha}-\frac{2}{(1-\gamma) \theta},
$$

which is positive by Assumption 1. In addition, $\frac{\partial g(C, C)}{\partial C_{L}}<0$ for all $C$. It now follows from the proof of Lemma 1 that $\widehat{C}(0,1)=\alpha$. QED

Proof of Lemma 2: We know that $C=\widehat{C}(\sigma, \xi)$ and hence we need to show that $\pi(\widehat{C}(\sigma, \xi), 0 ; \xi)=$ $\pi^{*}$. If not, then either $\pi(\widehat{C}(\sigma, \xi), 0 ; \xi)>\pi^{*}$ or $\pi(\widehat{C}(\sigma, \xi), 0 ; \xi)<\pi^{*}$. In the former case, parties have a dominant strategy to choose moderates and hence $\sigma=1$. Since $C>0$, it follows that by Bayes Rule $\rho=1$. But this implies that $\xi=0$ and hence that $C=\widehat{C}(\sigma, \xi)=0$. Thus, $\pi(C, 0 ; \xi)=\frac{1}{2}<\pi^{*}$ - a contradiction. In the latter case, parties have a dominant strategy to choose partisans and hence $\sigma=0$. In addition, (by assumption) we know that $\rho=0$. But this implies that $\xi=1$ and hence, from the properties of $\widehat{C}(\sigma, \xi)$, that $\pi(\widehat{C}(0,1), 0 ; 1)=1>\pi^{*}$ - a contradiction. QED

Proof of Formula for $\bar{\sigma}$ : By definition we know that

$$
\bar{\sigma}=\max \left\{\sigma \in[0,1]: \pi(\widehat{C}(\sigma, 1), 0 ; 1) \geq \pi^{*}\right\}
$$

Moreover, it is clear that $\widehat{C}(\sigma, 1)$ satisfies the first order condition

$$
\frac{\sigma \alpha \beta(1-2 x)}{2\left(\widehat{C}^{2}+\alpha^{2}\right)}+\frac{(1-\sigma) \beta(1-x-d)}{2 \alpha}=\frac{2}{\theta(1-\gamma)},
$$

which implies that

$$
\begin{aligned}
\frac{1}{2\left(\widehat{C}^{2}+\alpha^{2}\right)} & =\frac{2 \alpha-(1-\sigma) \beta \gamma \theta(1-x-d)}{\alpha(1-\gamma) \theta \sigma \alpha \beta(1-2 x)} \\
\widehat{C}^{2} & =\frac{\alpha^{2}(1-\gamma) \theta \sigma \beta(1-2 x)}{4 \alpha-2(1-\sigma) \beta \gamma \theta(1-x-d)}-\alpha^{2}
\end{aligned}
$$

We know that if $\bar{\sigma}<1$ then it must be the case that

$$
\widehat{C}(\bar{\sigma}, 1)=\alpha\left(2 \pi^{*}-1\right)
$$


or, equivalently,

$$
\widehat{C}^{2}=\alpha^{2}\left(2 \pi^{*}-1\right)^{2}
$$

It follows that if $\bar{\sigma}<1$, then it must be the case that

$$
\left(2 \pi^{*}-1\right)^{2}+1=\frac{(1-\gamma) \theta \sigma \beta(1-2 x)}{4 \alpha-2(1-\sigma) \beta \gamma \theta(1-x-d)}
$$

Solving for $\bar{\sigma}$, we obtain

$$
\begin{aligned}
\bar{\sigma} & =\frac{\left[\left(2 \pi^{*}-1\right)^{2}+1\right][\beta(1-\gamma) \theta(1-x-d)-4 \alpha]}{\beta(1-\gamma) \theta\left[(1-x-d)\left(\left(2 \pi^{*}-1\right)^{2}+1\right)-(1-2 x)\right]} \\
& =\frac{\left[\left(2 \pi^{*}-1\right)^{2}+1\right][\beta(1-\gamma) \theta(1-x-d)-4 \alpha]}{\beta(1-\gamma) \theta\left[(1-x-d)\left(2 \pi^{*}-1\right)^{2}+x-d\right]}
\end{aligned}
$$

This completes the proof of the formula. QED

Proof of Proposition 1: We know that $(\sigma, C, \xi, \rho)$ is a symmetric equilibrium if and only if $\sigma \in$ $[0, \bar{\sigma}], \xi=\widehat{\xi}(\sigma), C=\widehat{C}(\sigma, \widehat{\xi}(\sigma)), \rho \in \widehat{\rho}(\widehat{\xi}(\sigma))$ and

$$
\sigma=\frac{\rho}{\rho+(1-\rho)(1-\lambda(\widehat{C}(\sigma, \widehat{\xi}(\sigma))))}
$$

Thus, using the functional form for $\lambda$, there exists an equilibrium if and only if there exists $(\sigma, \rho) \in$ $[0, \bar{\sigma}] \times[0,1]$ such that $\rho \in \widehat{\rho}(\widehat{\xi}(\sigma))$ and

$$
\sigma=\frac{\rho(\widehat{C}(\sigma, \widehat{\xi}(\sigma))+\alpha)}{\rho \widehat{C}(\sigma, \widehat{\xi}(\sigma))+\alpha} .
$$

The first task is to understand the correspondence $\widehat{\rho}(\xi)$. It is the case that $\widehat{\rho}(0)=[\bar{\rho}, 1]$; $\widehat{\rho}(1)=[0, \underline{\rho}]$; and $\widehat{\rho}(\xi)=\{f(\xi)\}$ for all $\xi \in(0,1)$ where $f:[0,1] \rightarrow[\underline{\rho}, \bar{\rho}]$ is the function defined by:

$$
f(\xi)=\frac{\beta(3 x-1-d)-H^{-1}(\xi)}{\beta(x-d)} .
$$

For future reference, note that $f(0)=\bar{\rho}, f(1)=\underline{\rho}$, and that $f(\xi)$ is decreasing.

Now define the function $\varphi:[0, \bar{\sigma}] \rightarrow \Re$ as follows:

$$
\varphi(\sigma)=\sigma-\frac{f(\widehat{\xi}(\sigma))(\widehat{C}(\sigma, \widehat{\xi}(\sigma))+\alpha)}{f(\widehat{\xi}(\sigma)) \widehat{C}(\sigma, \widehat{\xi}(\sigma))+\alpha}
$$


It is the case that $\varphi(0)<0$. This is because $\widehat{\xi}(0) \in(0,1)$ and $f(\xi)>\underline{\rho}$ for all $\xi<1$. It is also the case that $\varphi(\sigma)$ is a continuously differentiable function that is increasing on $[0, \bar{\sigma}]$. To see the latter point, note that

$$
\varphi^{\prime}(\sigma)=1-\frac{\alpha\left[f^{\prime} \widehat{\xi}(\widehat{C}+\alpha)+f(1-f) \widehat{C}^{\prime}\right]}{(f(\widehat{\xi}(\sigma)) \widehat{C}(\sigma, \widehat{\xi}(\sigma))+\alpha)^{2}}
$$

and that both $f^{\prime} \widehat{\xi} \prime$ and $\widehat{C}^{\prime}$ are negative. For the latter, note that $\widehat{C}(\sigma, \widehat{\xi}(\sigma))$ must satisfy the equation $\pi(\widehat{C}(\sigma, \widehat{\xi}(\sigma)), 0 ; \widehat{\xi}(\sigma))=\pi^{*}$ and that increasing $\sigma$ must raise the effectiveness of campaign advertising. This means that the amount of campaign contributions necessary to achieve the probability $\pi^{*}$ falls.

Suppose now that $\varphi(\bar{\sigma}) \geq 0$. Then, there must exist a unique $\sigma^{*}$ such that $\varphi\left(\sigma^{*}\right)=0$. Letting $\rho^{*}=f\left(\widehat{\xi}\left(\sigma^{*}\right)\right)$, it is clear that $\rho^{*} \in \widehat{\rho}\left(\widehat{\xi}\left(\sigma^{*}\right)\right)$ and that

$$
\sigma^{*}=\frac{\rho^{*}\left(\widehat{C}\left(\sigma^{*}, \widehat{\xi}\left(\sigma^{*}\right)\right)+\alpha\right)}{\rho^{*} \widehat{C}\left(\sigma^{*}, \widehat{\xi}\left(\sigma^{*}\right)\right)+\alpha}
$$

Moreover, for every $\sigma \in\left[0, \sigma^{*}\right), \widehat{\xi}(\sigma)<\widehat{\xi}\left(\sigma^{*}\right)$ and hence $\rho \in \widehat{\rho}(\widehat{\xi}(\sigma))$ implies that

$$
\sigma<\frac{\rho(\widehat{C}(\sigma, \widehat{\xi}(\sigma))+\alpha)}{\rho \widehat{C}(\sigma, \widehat{\xi}(\sigma))+\alpha}
$$

Similarly, for every $\sigma \in\left(\sigma^{*}, \bar{\sigma}\right], \widehat{\xi}(\sigma)>\widehat{\xi}\left(\sigma^{*}\right)$ and hence $\rho \in \widehat{\rho}(\widehat{\xi}(\sigma))$ implies that

$$
\sigma>\frac{\rho(\widehat{C}(\sigma, \widehat{\xi}(\sigma))+\alpha)}{\rho \widehat{C}(\sigma, \widehat{\xi}(\sigma))+\alpha}
$$

Thus, there exists a unique symmetric equilibrium.

$$
\begin{aligned}
& \text { If } \varphi(\bar{\sigma})<0 \text {, then for every } \sigma \in[0, \bar{\sigma}), \widehat{\xi}(\sigma)<\widehat{\xi}(\bar{\sigma}) \text { and hence } \rho \in \widehat{\rho}(\widehat{\xi}(\sigma)) \text { implies that } \\
& \qquad \sigma<\frac{\rho(\widehat{C}(\sigma, \widehat{\xi}(\sigma))+\alpha)}{\rho \widehat{C}(\sigma, \widehat{\xi}(\sigma))+\alpha} .
\end{aligned}
$$

Thus, if there exists an equilibrium it must involve $\sigma=\bar{\sigma}$. The fact that $\varphi(\bar{\sigma})<0$ means that $\bar{\sigma}<1$ which in turn implies that $\widehat{\xi}(\bar{\sigma})=1$. It follows that $\widehat{\rho}(\widehat{\xi}(\bar{\sigma}))=[0, \underline{\rho}]$. Now choose $\rho^{*} \in(0, \underline{\rho})$ such that

$$
\bar{\sigma}=\frac{\rho^{*}(\widehat{C}(\bar{\sigma}, 0)+\alpha)}{\rho^{*} \widehat{C}(\bar{\sigma}, 0)+\alpha}
$$


Then, it is clear that $\left(\bar{\sigma}, \rho^{*}\right)$ satisfy the requirements of equilibrium and, moreover, since $\rho^{*}$ is uniquely defined, this is the only equilibrium.

This proves that there exists a unique symmetric equilibrium $\left(\sigma^{*}, C^{*}, \xi^{*}, \rho^{*}\right)$. The remainder of the proposition follows from noting that $\sigma^{*}>0$ and that $\pi\left(C^{*}, 0 ; \xi^{*}\right)=\pi^{*} \in\left(\frac{1}{2}, 1\right)$. QED

Proof of Proposition 2: We claim that $(0, L, 1,0)$ is the unique symmetric equilibrium. It is clear that it is an equilibrium, so we need only show that it is unique. We know that if $(\sigma, C, \xi, \rho)$ is a symmetric equilibrium with contribution limit $L$, then it must be the case that $C \leq L$ and $\xi \leq 1$. This implies that $\pi(C, 0, \xi)<\pi^{*}$ which implies that $\sigma=0$. This, in turn, implies that $\rho=0$ and hence that $\xi=1$. This, in turn implies that $C=L$. Expected campaign spending in this equilibrium must be zero, because interest groups only give to moderates and parties always select partisans. QED

Proof of Lemma 3: We first claim that $\pi(C, 0 ; \xi)=\pi^{*}$. If not, then either $\pi(C, 0 ; \xi)>\pi^{*}$ or $\pi(C, 0 ; \xi)<\pi^{*}$. In the former case, $\sigma=1$. Since $C>0$, it follows that by Bayes Rule $\rho=1$. But this implies that $\xi=0$ and hence that $\pi(C, 0 ; \xi)=\frac{1}{2}<\pi^{*}$ - a contradiction. In the latter case, $\sigma=0$. In addition, (by assumption) we know that $\rho=0$. But this implies that $\xi=1$ and hence that $C=L$ and that $\pi(C, 0 ; 1)=\pi(L, 0 ; 1)$. But since $L$ is at least as big as $\alpha\left[2 \pi^{*}-1\right]$, we have that $\pi(L, 0 ; 0) \geq \pi^{*}-$ a contradiction.

It remains to show that $C=L$. Letting $\left(\sigma^{*}, C^{*}, \xi^{*}, \rho^{*}\right)$ be the laissez-faire equilibrium, we know that $\pi(C, 0 ; \xi)=\pi\left(C^{*}, 0 ; \xi^{*}\right)$ where $C \leq L<C^{*}$. It follows that $\xi>\xi^{*}$. If $\sigma=0$, it follows immediately that $C=L$ since if the constraint were not binding the equilibrium contribution level would be $\widehat{C}(0, \xi)$ which must exceed $C^{*}$ and hence $L$. If $\sigma>0$ then

$$
\sigma=\frac{\rho}{\rho+(1-\rho)(1-\lambda(C))}
$$

But we know that $\rho<\rho^{*}$ and that $C<C^{*}$ which implies that $\sigma<\sigma^{*}$. This means that $C=L$ since if the constraint were not binding the equilibrium contribution level would be $\widehat{C}(\sigma, \xi)$ which 
must exceed $C^{*}$ and hence $L$. QED

Proof of Proposition 3: We know that if $(\sigma, C, \xi, \rho)$ is a symmetric equilibrium then $\xi=\widetilde{\xi}(L)$ and $C=L$. If $L$ is bigger than $\alpha\left[2 \pi^{*}-1\right]$, then we know that $\xi \in(0,1)$ which implies that $\rho=$ $f(\widetilde{\xi}(L))$ (where $f$ is as defined in Proposition 1) and that

$$
\sigma=\frac{f(\widetilde{\xi}(L))(L+\alpha)}{f(\widetilde{\xi}(L)) L+\alpha}
$$

This is the unique symmetric equilibrium.

If $L$ equals $\alpha\left[2 \pi^{*}-1\right]$, then we know that $\xi=1$ which implies that $\rho \in[0, \underline{\rho}]$. Letting

$$
\widehat{\sigma}(\rho)=\frac{\rho \alpha 2 \pi^{*}}{\rho \alpha\left[2 \pi^{*}-1\right]+\alpha}
$$

the set of equilibria are $\{(\widehat{\sigma}(\rho), L, 0, \rho): \rho \in[0, \underline{\rho}]\}$.

It remains to show that both the probability that parties select moderates and expected campaign spending are decreasing in the size of the limit. Let $\sigma^{*}(L)$ denote the probability that parties select moderates with limit $L>\alpha\left[2 \pi^{*}-1\right]$. Then

$$
\sigma^{*}(L)=\frac{f(\widetilde{\xi}(L))(L+\alpha)}{f(\widetilde{\xi}(L)) L+\alpha}
$$

Differentiating, we obtain

$$
\begin{aligned}
\frac{d \sigma^{*}(L)}{d L} & =\frac{(f L+\alpha)\left[f+f^{\prime} \widetilde{\xi}^{\prime}(L+\alpha)\right]-\left(f+f^{\prime} \widetilde{\xi}^{\prime} L\right) f(L+\alpha)}{(f L+\alpha)^{2}} \\
& =\frac{f^{2} L+\alpha f+\alpha f^{\prime} \widetilde{\xi}^{\prime}(L+\alpha)-f^{2}(L+\alpha)}{(f L+\alpha)^{2}} \\
& =\frac{\alpha f(1-f)+\alpha f^{\prime} \widetilde{\xi}^{\prime}(L+\alpha)}{(f L+\alpha)^{2}}
\end{aligned}
$$

This is positive since $f^{\prime} \widetilde{\xi}^{\prime}>0$, which proves that $\sigma^{*}(L)$ is decreasing on $\left(\alpha\left[2 \pi^{*}-1\right], C^{*}\right]$. With limit $L=\alpha\left[2 \pi^{*}-1\right]$, the probability that parties select moderates is no greater than

$$
\frac{\rho \alpha 2 \pi^{*}}{\underline{\rho} \alpha\left[2 \pi^{*}-1\right]+\alpha}
$$


But for all $L>\alpha\left[2 \pi^{*}-1\right]$, we have that

$$
\sigma^{*}(L)=\frac{f(\widetilde{\xi}(L))(L+\alpha)}{f(\widetilde{\xi}(L)) L+\alpha}>\frac{\rho(L+\alpha)}{\underline{\rho} L+\alpha}>\frac{\underline{\rho} \alpha 2 \pi^{*}}{\underline{\rho} \alpha\left[2 \pi^{*}-1\right]+\alpha} .
$$

Expected campaign spending with limit $L>\alpha\left[2 \pi^{*}-1\right]$ is given by

$$
E^{*}(L)=2 \sigma^{*}(L) L
$$

This is clearly increasing in $L$. With limit $L=\alpha\left[2 \pi^{*}-1\right]$, expected campaign spending is no greater than

$$
2\left(\frac{\underline{\rho} \alpha 2 \pi^{*}}{\underline{\rho} \alpha\left[2 \pi^{*}-1\right]+\alpha}\right)\left(\alpha\left[2 \pi^{*}-1\right]\right),
$$

which is smaller than $E^{*}(L)$ for all $L>\alpha\left[2 \pi^{*}-1\right]$. QED

Proof of Proposition 4: (i) Let $\left(\sigma^{*}(\gamma), C^{*}(\gamma), \xi^{*}(\gamma), \rho^{*}(\gamma)\right)$ denote the laissez-faire equilibrium when the fraction of moderates is $\gamma$ and let $E^{*}(\gamma)$ denote expected campaign spending. Further, let $W\left(\sigma, \pi^{*}, E, \gamma\right)$ denote aggregate welfare with the pair $(E, \sigma)$. Recall that

$$
\bar{\sigma}=\max \left\{1, \frac{\left[\left(2 \pi^{*}-1\right)^{2}+1\right][\beta(1-\gamma) \theta(1-x-d)-4 \alpha]}{\beta(1-\gamma) \theta\left[(1-x-d)\left(2 \pi^{*}-1\right)^{2}+x-d\right]}\right\}
$$

and let $\widehat{\gamma}$ be the solution to the equation

$$
\frac{\underline{\rho} 2 \pi^{*}}{\left[\underline{\rho} 2 \pi^{*}+1-\underline{\rho}\right]}=\frac{\left[\left(2 \pi^{*}-1\right)^{2}+1\right][\beta(1-\gamma) \theta(1-x-d)-4 \alpha]}{\beta(1-\gamma) \theta\left[(1-x-d)\left(2 \pi^{*}-1\right)^{2}+x-d\right]} .
$$

Observe that since $\underline{\rho}>0$ the left hand side is positive, less than 1 and is independent of $\theta$. The right hand side is decreasing in $\gamma$ and less than or equal to zero when $\frac{1-\gamma}{2} \leq \frac{2 \alpha}{\beta \theta(1-x-d)}$. Thus, $\widehat{\gamma}<1-\frac{4 \alpha}{\beta \theta(1-x-d)}$. Now let

$$
\bar{\gamma}=\max \left\{\frac{4 \alpha(x-d)}{\beta(1-2 x)(1-x-d)}, \widehat{\gamma}\right\}
$$

We claim that $\bar{\gamma}$ is smaller than $1-\frac{4 \alpha}{\beta \theta(1-x-d)}$. To prove this, we need to show that

$$
\frac{4 \alpha(x-d)}{\beta(1-2 x)(1-x-d)}<1-\frac{4 \alpha}{\beta \theta(1-x-d)} .
$$


Rearranging, this becomes

$$
\frac{4 \alpha}{\beta(1-x-d)}\left[\frac{(x-d)}{(1-2 x)}+\frac{1}{\theta}\right]<1
$$

or, equivalently,

$$
\frac{4 \alpha[\theta(x-d)+1-2 x]}{\theta(1-2 x)(1-x-d)}<\beta
$$

This holds by hypothesis.

When $\gamma>\bar{\gamma}$, the laissez-faire equilibrium is a boundary equilibrium since, by construction, $\frac{\underline{\rho} 2 \pi^{*}}{\left.\underline{\rho} \underline{\rho} \pi^{*}+1-\underline{\rho}\right]}>\bar{\sigma}$. Thus, $\left(C^{*}(\gamma), \xi^{*}(\gamma)\right)=\left(\alpha\left(2 \pi^{*}-1\right), 0\right)$ and

$$
\left(\sigma^{*}(\gamma), \rho^{*}(\gamma)\right)=\left(\bar{\sigma}, \frac{\bar{\sigma}}{2 \pi^{*}-\left(2 \pi^{*}-1\right) \bar{\sigma}}\right)
$$

It follows that imposing any contribution limit will lead to both zero expected campaign spending and a zero probability of parties selecting moderates (i.e., the pair $(E, \sigma)=(0,0))$. Thus, to complete the proof all we need to do is to show that welfare at the laissez-faire equilibrium $W\left(\sigma^{*}(\gamma), \pi^{*}, E^{*}(\gamma), \gamma\right)$ is greater than $W\left(0, \pi^{*}, 0, \gamma\right)$. The way we do this is to first show that $\left(E^{*}(\gamma), \sigma^{*}(\gamma)\right)$ lies on the line joining $(0,0)$ and $(\widehat{E}, \widehat{\sigma})$ in Figure 1. We then show that any point on this curve generates a higher level of welfare than does $(0,0)$ under the stated condition.

Since $\rho^{*}(\gamma)=\frac{\bar{\sigma}}{2 \pi^{*}-\left(2 \pi^{*}-1\right) \bar{\sigma}}$, we know that

$$
\bar{\sigma}=\frac{\rho^{*}(\gamma) 2 \pi^{*}}{\rho^{*}(\gamma)\left(2 \pi^{*}-1\right)+1}
$$

and that $\rho^{*}(\gamma) \leq \underline{\rho}$. It follows that the laissez-faire equilibrium belongs to the set

$$
\Phi=\left\{\left(\frac{\rho 2 \pi^{*}}{\rho\left(2 \pi^{*}-1\right)+1}, \alpha\left(2 \pi^{*}-1\right), 0, \rho\right): \rho \in[0, \underline{\rho}]\right\}
$$

which is none other than the set of equilibria that can arise with the contribution limit $L=$ $\alpha\left(2 \pi^{*}-1\right)$. Corresponding to any point in the set $\Phi$ is an expected campaign spending level and a probability that parties select moderates $(E(\rho), \sigma(\rho))$. It is straightforward to show that

$$
(E(\rho), \sigma(\rho))=\left(\frac{\rho \alpha\left(4 \pi^{*}\right)\left(2 \pi^{*}-1\right)}{\left(\rho\left(2 \pi^{*}-1\right)+1\right)}, \frac{\rho\left(2 \pi^{*}\right)}{\left(\rho\left(2 \pi^{*}-1\right)+1\right)}\right) .
$$


Varying $\rho$ between $[0, \underline{\rho}]$ yields the curve joining $(0,0)$ to $(\widehat{E}, \widehat{\sigma})$ in Figure 1 . An explicit description of the curve may be obtained by noting that

$$
E^{-1}(E)=\frac{E}{\left(2 \pi^{*}-1\right)\left(\alpha 4 \pi^{*}-E\right)}
$$

and hence the curve is described by the function

$$
\sigma\left(E^{-1}(E)\right)=\frac{E}{2 \alpha\left(2 \pi^{*}-1\right)}
$$

This function is linear, establishing that the curve has the shape depicted in Figure 1.

We now show that any point on the curve $(\neq(0,0))$ yields a higher level of welfare than $(0,0)$ under the stated condition. Suppose that it were the case that $(\widehat{E}, \widehat{\sigma})$ lay on a higher social indifference curve than $(0,0)$. Then, by the convexity of the social indifference curves, so must any other point $(\neq(0,0))$ on the curve. To prove that $(\widehat{E}, \widehat{\sigma})$ lies on a higher social indifference curve than $(0,0)$, it is enough to show that the slope of the social indifference curve at $(\widehat{E}, \widehat{\sigma})$ is no greater than the slope of the line segment. The above analysis implies that the latter is given by:

$$
\frac{d \sigma}{d E}=\frac{1}{2 \alpha\left(2 \pi^{*}-1\right)}
$$

The indifference curves have slope

$$
\frac{d \sigma}{d E}=\frac{1}{\gamma \partial W_{M}\left(\widehat{\sigma}, \pi^{*}\right) / \partial \sigma}
$$

Thus, the required condition is that

$$
\gamma \partial W_{M}\left(\widehat{\sigma}, \pi^{*}\right) / \partial \sigma \geq 2 \alpha\left(2 \pi^{*}-1\right)
$$

We must now solve explicitly for the derivative $\partial W_{M}\left(\widehat{\sigma}, \pi^{*}\right) / \partial \sigma$. Note first that we may write:

$$
W_{M}\left(\sigma, \pi^{*}\right)=-\frac{\sigma^{2}}{2} \psi_{M M}-\frac{(1-\sigma)^{2}}{2} \psi_{P P}-\sigma(1-\sigma) \psi_{P M},
$$


where

$$
\begin{aligned}
\psi_{P P} & =\frac{\beta(1-x-d)}{2}+\frac{3 \beta(x-d)}{2}, \\
\psi_{M M} & =\frac{\beta(1-2 x)}{2}, \text { and } \\
\psi_{P M} & =\left(\pi^{*}\right)^{2} \beta(1-2 x)+\left(1-\pi^{*}\right)^{2} \beta(1-x-d)+\left(1-\left(\pi^{*}\right)^{2}\right) \beta(x-d) .
\end{aligned}
$$

Differentiating, we obtain

$$
\frac{\partial W_{M}\left(\sigma, \pi^{*}\right)}{\partial \sigma}=\psi_{P P}-\psi_{P M}-\sigma\left(\psi_{P P}+\psi_{M M}-2 \psi_{P M}\right)
$$

It is straightforward to show that $\psi_{P P}+\psi_{M M}>2 \psi_{P M} ; \psi_{P P}>\psi_{P M}$; and $\psi_{P M}>\psi_{M M}$. These inequalities imply that $\frac{\partial W_{M}\left(\sigma, \pi^{*}\right)}{\partial \sigma}$ is positive but decreasing in $\sigma$. It follows therefore, that

$$
\frac{\partial W_{M}\left(\widehat{\sigma}, \pi^{*}\right)}{\partial \sigma}>\psi_{P M}-\psi_{M M}
$$

Moreover, since $x-d>1-2 x$

$$
\psi_{P M}-\psi_{M M}>\frac{\beta(1-2 x)}{2}
$$

Thus,

$$
\gamma \frac{\partial W_{M}\left(\widehat{\sigma}, \pi^{*}\right)}{\partial \sigma}>\frac{\gamma \beta(1-2 x)}{2}
$$

Next observe that, using the definition of $\pi^{*}$, we have:

$$
2 \alpha\left(2 \pi^{*}-1\right)=\frac{2 \alpha(x-d)}{1-x-d}
$$

Thus, a sufficient condition for $\gamma \partial W_{M}\left(\widehat{\sigma}, \pi^{*}\right) / \partial \sigma \geq 2 \alpha\left(2 \pi^{*}-1\right)$ is that:

$$
\frac{\gamma \beta(1-2 x)}{2} \geq \frac{2 \alpha(x-d)}{1-x-d}
$$

This is implied by the definition of $\bar{\gamma}$ and the fact that $\gamma>\bar{\gamma}$.

(ii) There are two cases to consider. Suppose first that $\widehat{\gamma}$ as defined above is less than or equal to 0 . Then, for all $\gamma$, the laissez-faire equilibrium is a boundary equilibrium in which $C^{*}(\gamma)=$ 
$\alpha\left(2 \pi^{*}-1\right)$. As shown above, this means that the pair $\left(E^{*}(\gamma), \sigma^{*}(\gamma)\right)$ lies on the line connecting $(0,0)$ and $(\widehat{E}, \widehat{\sigma})$ depicted in Figure 1. Moreover, as we decrease $\gamma$ towards $0,\left(E^{*}(\gamma), \sigma^{*}(\gamma)\right)$ moves continuously up the curve reflecting the fact that $\bar{\sigma}$ is increasing. Now let

$$
\lim _{\gamma \rightarrow 0}\left(E^{*}(\gamma), \sigma^{*}(\gamma)\right)=(\widetilde{E}, \widetilde{\sigma})
$$

Then,

$$
\lim _{\gamma \rightarrow 0} W\left(\sigma^{*}(\gamma), \pi^{*}, E^{*}(\gamma), \gamma\right)=-\widetilde{E}-\frac{\beta(1-2 d)}{2}<-\frac{\beta(1-2 d)}{2}
$$

Since for all $\gamma, W\left(0, \pi^{*}, 0, \gamma\right)=-\frac{\beta(1-2 d)}{2}$, there must exist $\underline{\gamma}>0$ such that $W\left(0, \pi^{*}, 0, \gamma\right)>$ $W\left(\sigma^{*}(\gamma), \pi^{*}, E^{*}(\gamma), \gamma\right)$ for all $\gamma \in(0, \underline{\gamma})$. Since $(0,0)$ can be achieved by imposing any contribution limit $L<\alpha\left(2 \pi^{*}-1\right)$, the result follows.

If $\widehat{\gamma}$ is greater than 0 , then for all $\gamma \in(0, \widehat{\gamma})$, the laissez-faire equilibrium is an interior equilibrium in which $C^{*}(\gamma)>\alpha\left(2 \pi^{*}-1\right)$. As we decrease $\gamma$ in this range, the equilibrium level of contributions increases reflecting the greater size of the interest groups. In addition, the probability that parties select moderates increases. This translates into a continuous rightward move of the pair $\left(E^{*}(\gamma), \sigma^{*}(\gamma)\right)$. Now let

$$
\lim _{\gamma \rightarrow 0}\left(E^{*}(\gamma), \sigma^{*}(\gamma)\right)=(\widetilde{E}, \widetilde{\sigma})
$$

Then,

$$
\lim _{\gamma \rightarrow 0} W\left(\sigma^{*}(\gamma), \pi^{*}, E^{*}(\gamma), \gamma\right)=-\widetilde{E}-\frac{\beta(1-2 d)}{2}<-\frac{\beta(1-2 d)}{2}
$$

and it again follows that there must exist $\underline{\gamma}>0$ such that $W\left(0, \pi^{*}, 0, \gamma\right)>W\left(\sigma^{*}(\gamma), \pi^{*}, E^{*}(\gamma), \gamma\right)$ for all $\gamma \in(0, \underline{\gamma})$. QED 


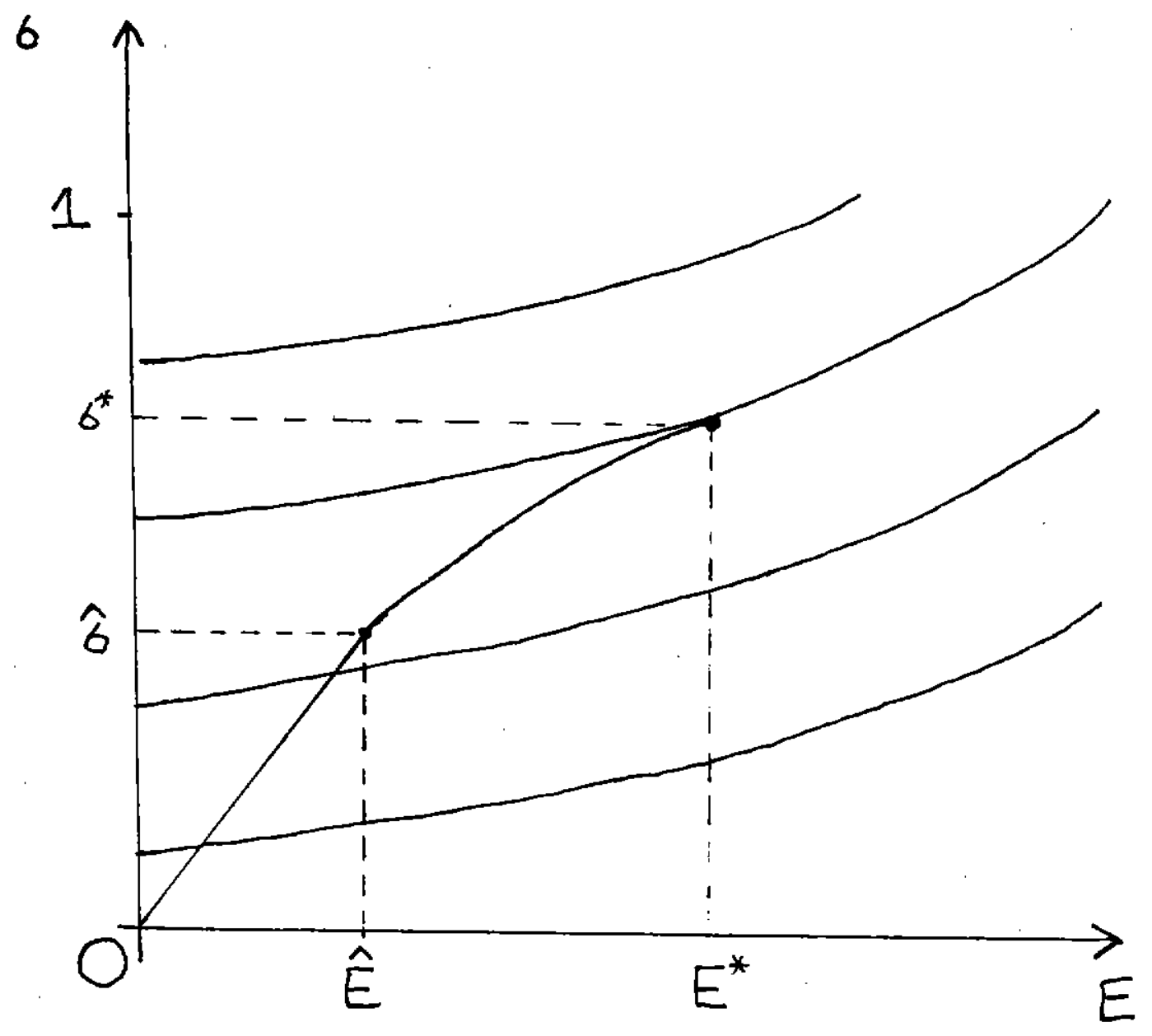

Figure 1 